\title{
XV.
}

\section{Bericlit über die städtische Cholera-Heilanstalt No. III zu Berlin während der Epidemie des Jahres 1866.}

\author{
Von Dr. M. Goldbaum, dirigirendem Arzte.
}

$D_{\text {i }}$

diessjährige Epidemie ist von den eilf Epidemien, welche Berlin bis jetzt heimgesucht, die verbreiteteste gewesen; sie hat an Extensităt die bisher grösste Epidemie des Jahres 1849 weit überbolt. Von den vier von Seiten der Sanitätscommission errichteten Cholera-Heilanstalten ist die Leitung der III. im nördlichen Theil der Stadt (Tiekstrasse 14) gelegenen mir anvertraut worden.

Das Lazareth bildete die Ecke der Tiek - und Borsigstrasse, lag also von zwei Seiten frei, dem Luftzuge und den Sonnenstrahlen hinlänglich exponirt, war aber nicht von beiden Häuserreihen getrennt. Das Lazareth war von den städtischen Behörden, soweit der beabsichtigte Zweck und die rasche Herstellung desselben zuliess, mit gewohnter Munificenz eingerichtet*). In dasselbe wurden vom 14. Juli bis zum 20. October 540 Cholerakranke aufgenommen.

Nach Abzug der 51 todt u. sterbend Eingebrachten 51

bleiben 489 Personen.

Hiervon wurden geheilt entlassen . . . 253 oder $51,74 \mathrm{pCt}$. und starben . . . 236 oder 48,26 pCt.

Einschliesslich der 51 todt $u$. sterbend Eingebrachten waren: Männer . . . . 227 davon genasen 104 oder 45,81 pCt. und starben 123 oder 54,18 -

*) Als Assistenzärzte fungirten im Lazareth: die Herren DDr. Stappenbeck, Ahronheim und Bruberger, welche mit grosser Hingebung und angestrengtester Thätigkeit der Krankenpflege oblagen, aber auch mit eben so grossem Eifer und regem Interesse die wissenschaftlichen Fragen verfolgten. Letzterer, ein Schüler des Hrn. Kühn e, hat besonders die chemischen und mikroskopischen Arbeiten ausgeführt. Wir baben ihm daher dieses Material zur zusammenhängenden Bearbeitung im nachstehenden Aufsațe überlassen. 
Weiber . . . 235 davon genasen 109 oder 46,39 pCt.

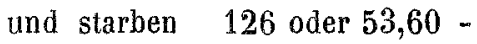

Knaben bis incl. 10 Jahre

Mådchen
37 davon genasen 19 oder 51,35 und starben 18 oder 48,64 -

41 davon genasen 21 oder 51,22 und starben 20 oder 48,77 -

Hieraus ergibt sich wiederum, wie in früheren Epidemien, das weit günstigere Sterblichkeitsverbältniss in den Choleralazarethen als in der Stadt. Nach den von der Sanitätscommission veröffentlichten Zahlen ist das Sterblichkeitverhältniss in der Stadt 66 pCt. gewesen, während in unserem Lazareth, selbst einschliesslich der todt Eingebrachten nur 53 pCt. gestorben sind. Nach Abzug der in den 4 Lazarethen behandelten Kranken dürfte sich demnach die Sterblichkeit in der Stadt auf über 70 pCt. stellen.

Einfluss des Alters a f Genesung und Sterblichkeit.

\begin{tabular}{|c|c|c|c|c|c|c|c|c|c|c|c|}
\hline \multicolumn{5}{|c|}{ Alter } & $\begin{array}{l}\text { männ- } \\
\text { liche }\end{array}$ & $\begin{array}{l}\text { weib- } \\
\text { liche }\end{array}$ & Summa & Genesen & Gestorben & \multicolumn{2}{|l|}{$\begin{array}{l}\text { Procente } \\
\text { der Gestor- } \\
\text { benen }\end{array}$} \\
\hline Bis inclu & 1 & Jahr & & & 8 & 4 & 12 & 5 & 7 & $58,33 \mathrm{pC}$ & \\
\hline $1-10$ & abre & incl & lus. & & 28 & 37 & 65 & 35 & 30 & $46,15-$ & \\
\hline $10-20$ & - & . & · & . & 37 & 34 & 71 & 41 & 30 & 42,25 & \\
\hline $20-30$ & - & & . & - & 56 & 82 & 138 & 81 & 57 & 41,30 & \\
\hline $30-40$ & - & $\cdot$ & . & . & 51 & 61 & 112 & 48 & 64 & $57,14-$ & - \\
\hline $40-50$ & - & . & . & - & 42 & 24 & 66 & 22 & 44 & 66,66 - & - \\
\hline $50-60$ & - & . & . & . & 27 & 13 & 40 & 14 & 26 & 65 & - \\
\hline $60-70$ & - & . & . & $=$ & 11 & 14 & 25 & 5 & 20 & 80 & - \\
\hline $70-90$ & - & . & . & . & 4 & 7 & 11 & $\mathbf{3}$ & 8 & 72,7 & - \\
\hline
\end{tabular}

Hiernach wird mit jedem Jahrzehnt die Sterblichkeit geringer bis zum 30. Jahre und höher bis zum 70. Auffallend günstig ist die Sterblichkeit in den höchsten Altersstufen; während sie in früheren Epidemien $90-100$ pCt. betrug, starben in unserem Lazareth nur $72 \mathrm{pCt}$; ebenso günstig ist das Verhältniss vom 20.-30. Lebensjahre; während in früheren Jahren $45-54$ pCt. erlagen, starben bei uns nur 41 pCt. Hingegen gestaltele sich die Sterblichkeit im 1. Lebensjahre ungünstiger als in früheren Epidemien.

Die Dauer des Aufenthaltes in der Heilanstalt war: 
von 1-4 Stunden 56 Indiv., von denen 0 genesen u. 56 gestorben

$-4-8-44-0$ - 0 - 44 -

$-8-12-35-\quad-\quad 0 \quad-35$ -

- 12 St.bis 3Tage $121-\quad$ - $26-95$ -

- 3-6 Tagen 108 - $\quad-74-34$ -

- 6-14 Tagen 141 - $-120-21$ -

- 2-4 Wochen 29- - 27 - 2 -

uiber 4 Wochen

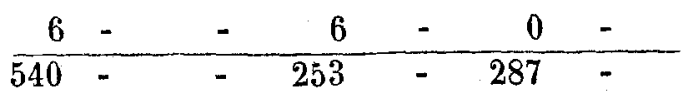

Hieraus lässt sich kein Schluss auf die wirkliche Dauer der Krankheit machen, da schon eine geringfügige Injectionswunde die Reconvalescenz erheblich verlängerte.

Insoweit man aus der Beschaffenheit des Pulses auf den Grad der Krankheit in den einzelnen Fällen schliessen darf, ist es von Wichtigkeit, sämmtliche Fälle nach der Beschaffenheit des Pulses bei der Aufnahme zu klassificiren, um so einen Anhaltspunkt für den weiteren Verlauf zu erhalten.

1) Von 223 bei der Aufnahme pulslosen Fällen sind 41 oder 18,3 pCt. genesen und 182 oder 81,6 pCt. gestorben. Nach Abzug der 51 todt und sterbend Eingebrachten, bei denen ein therapeutischer Eingriff gar nicht erst versucht worden ist, reducirt sich die Zahl der pulslosen Fälle auf 172, von denen also 41 oder 23,9 pCt. genasen, und 131 oder 76,0 pCt. gestorben.

2) Mit verschwindendem pulse sind in die Anstalt 110 aufgenommen, von denen 57 oder 51,8 pCt. genesen, und 53 oder 48,1 pCt. gestorben sind.

3) Bei 165 Fällen war der Puls klein und schwach, von denen 117 oder 70,9 pCt. genesen, und 48 oder 29 pCt. gestorben sind.

4) Mit noch gutem Pulse sind 42 Fälle aufgenommen, von denen 37 oder $88 \mathrm{pCt}$. genesen und 5 oder $11,9 \mathrm{pCt}$. gestorben sind.

Wir haben obige Klassification mit Absicht beibehalten, um die gegenwärtige Epidemie mit der letzten des Jahres 1855, wie sie sich in den Lazarethen manifestirte, vergleichen zu können*).

*) In den Epidemien der Jahre 1853, 1854 und 1855 habe ich in der CholeraHeilanstalt Wallstrasse 55 unter der umsichtigen Leitung des Herrn GeheimMedicinalrath Dr. Ed. Müller als Assistenzarzt fungirt. Ich werde daher 


\begin{tabular}{c|c|c|c|c|c|c|c|c}
\hline $\begin{array}{c}\text { Es waren } \\
\text { in der } \\
\begin{array}{c}\text { Epidemie } \\
\text { des Jahres: }\end{array}\end{array}$ & $\begin{array}{c}\text { Puls- } \\
\text { lose } \\
\text { Fälle }\end{array}$ & $\begin{array}{c}\text { Fälle mit } \\
\text { hiervon } \\
\text { starben }\end{array}$ & $\begin{array}{c}\text { Fälle mit } \\
\text { dendem } \\
\text { Pulse }\end{array}$ & $\begin{array}{c}\text { kleinem } \\
\text { und } \\
\text { pCt. }\end{array}$ & $\begin{array}{c}\text { favon } \\
\text { schwarbch. } \\
\text { Pulse }\end{array}$ & $\begin{array}{c}\text { Fälle } \\
\text { mit } \\
\text { starben }\end{array}$ & $\begin{array}{c}\text { gutem } \\
\text { Pulse }\end{array}$ & $\begin{array}{c}\text { hiervon } \\
\text { starben }\end{array}$ \\
\hline 1855 & 157 & 92,9 & 93 & 63 & 112 & 37 & 80 & 10 \\
1866 & 223 & 81,6 & 110 & 48,1 & 117 & 29 & 42 & 11,9
\end{tabular}

Hieraus geht hervor, dass trotz der überwiegenden Anzahl der asphyctischen Fälle, die in der letzten Epidemie im Vergleiche zu der vorletzten in's Lazareth eingeliefert worden, dennoch das Mortalitätsverhältniss 1866 in den vollkommen asphyctischen Fällen um 11 pCt., bei den im Uebergange zur Asphyxie begriffenen Fällen um 15 pCt. geringer war als 1855 .

Wir sind weit entfernt, dieses auffallende Ergebniss etwa unserer diessjährigen Behandlung zuzuschreiben, sondern vielmehr dem milderen Charakter der gegenwärtigen Epidemie; wie sie sich in unserem Lazarethe darstellte. Im Lazareth des Jahres 1855 gehörten die enterischen oder besser transsudativen Fälle zu den grossen Seltenheiten, während 1866 eine grosse Anzahl von asphyctischen Fällen mit enormen Transsudationen verliefen. Im Jahre 1853, wo die transsudativen Fälle auch häufiger waren, sind im Lazareth von 135 prilslosen Fällen 112 gestorben, also $83 \mathrm{pCt}$. Die Transsudationen aber sind, wie wir später ausführlich sehen werden, für den Verlauf sowohl der Asphyxie als des späteren Typhoids durchaus günstig. Es trat daher auch das typhoide Stadium viel milder auf, als im Jahre 1855, wo die ächt urämischen Fälle viel häufiger als in der letzten Epidemie waren. Wir kommen beim Typhoid ausfübrlich darauf zurück.

Die Verbreitungsweise und Aetiologie der Cholera ist in neuerer Zeit durch die Anschaungen Pettenk ofer's dem Urtheile und der Beobachtung des Hospitalarztes fast ganz entrückt. Wir kennen von den uns überlieferten Patienten weder den ort der Erkrankung in Bezug auf die Bodenverhältnisse noch die

mit gütiger Erlaubniss meines damaligen Chefs auf die Beobachtungen in jenen Lazarethen in dieser Arbeit recurriren, zumal die hier entwickelten Thatsachen meist schon damals beobachtet worden sind. 
Schädlichkeit, welche local auf den Ausb̂ruch der Krankbeit influirt haben könnte. Wir sind lediglich auf die vagen und im höchsten Grade unzuverlässigen Angaben der Patienten angewiesen, die nicht werth sind registrirt zu werden*). Nur die aus ejgener Anschauung geflossenen Angaben der Aerzte haben einen Werth. Wir wollen daher nur über die Verbreitung der Cholera in unserer Heilanstalt sprechen.

Obgleich in den Nachbarhäusern die Cholera sehr stark wüthete, sind in unserem Hause von dem sehr starken Personal nur zwei Todesfälle vorgekommen, und $z$ war in einem $Z$ wischenraume von vier Wochen. Die erste im Waschhause beschäftigt, Gravida im 6. Monat, starb nachdem sie die Cholera und einen Abort überstanden, im Puerperium. Die zweite, eine ängstliche Köchin, die weder mit Cholerakranken noch mit Dejectionen derselben in irgend welche Berührung kam, starb in der Asphyxie nach 10 Stunden. Die Herren Aerzte sowie die Wärter, welche Tag und Nacht in der angestrengtesten Thätigkeit sich mit den Patienten und Leichen nach allen Richtungen hin beschäftigten, erstere auch mit genauen Messungen, chemischen und mikroskopischen Untersuchungen der Dejectionen, sind von der Cholera ganz verschont geblieben. Auch ist zu erwähnen, dass eine in unserem Hause verbliebene zahlreiche Familie, die von Seiten der 'Behörde nicht hat exmittirt werden können, obgleich nur durch einen Verschlag vom eigentlichen Lazareth getrennt, dennoch keine Erkrankungen aufzuweisen hat.

Bei den in's Lazareth Eingelieferten hielten die gruppenweisen Erkrankungen ziemlich gleichen Schritt mit den vereinzelt in den Familien vorgekommenen Fällen, insoweit es sich nach den Angaben der Kranken selbst mit Sicherheit constatiren liess.

In der Absicht, der Contagiositätslehre Pettenkofer's näher $\mathrm{zu}$ treten, haben wir im Lazareth von vorn herein Infections versuche an Thieren angestellt, um die Versuche von Thiersch ${ }^{* *}$ )

*) Wir haben in früheren Jahren bei einer Arrestantin des Arbeitshauses ihre genaue und scheinbar glaubwürdige Angabe registrirt, sie habe die kleidungsstïcke einer an der Cholera Verstorbenen angezogen. Die genaueste Untersuchung ergab die Unwahrheit des ganzen Thatbestandes, und dennoch nahm sie die Lüge mit ins Grab. Einer Anderen, die Cholerawäsche gewaschen haben wollte, konnte die Unwahrheit mit Sicherheit nachgewiesen werden.

${ }^{* *}$ ) Infectionsversuche an Thieren, München, 1866 . 
zu prüfen. Da wir nur negative Resultate erhalten haben, fast analog den bereits von verschiedenen Seiten veröffentlichten*), so können wir uns kurz fassen.

Nachdem wir ohne Erfolg einen mittelgrossen Hund längere Zeit im geschlossenen Raume an der Dejectionsgrube haben befestigen lassen, so dass er nur diese mit Dejectionen inficirte Luft hat einathmen können, haben wir demselben frische und ältere Dejectionen unzenweise wiederholt in den Magen gebracht, später dieselben in Quantitäten von 3-4 Drachmen wiederholt subcutan injicirt. Nach allen Versuchen trat beim Thiere ein fieberhafter Zustand ein (sehr heisse Schnauze, frequente Respiration), zuweilen mehrere, aber fäculente Stühle. Dieses Unwohlsein hielt bei allen Thieren nur bis zum nächsten Morgen an. Nachdem wir nunmehr diesen Hund sechs Wochen, ohne ihn zu ferneren Versuchen zu benutzen, als Wächter im Hause behielten, starb er zum Schlusse des Lazareths an Convulsionen, ohne dass wir bei der Section eine Spur von Cholera haben entdecken können. Dasselbe negative Resultat ergaben unsere Versuche an Kaninchen, von denen noch vier beim Schlusse des Lazaretbs munter umhersprangen.

Wir sind daher zu der Annahme berechtigt, dass weder frische noch in Zersetzung übergegangene Dejectionen bei Thieren Cholera-Erscheinungen hervorzurufen vermögen. Die Erkrankungen der Thiere nach den Infectionsversuchen glauben wir vielmehr mit Stich**) der putriden Infection zuschreiben zu müssen.

Das auffallende Verhältniss des Wechselfiebers zur Cholera haben wir mehrfach beobachtet in Fällen, bei denen nach einem leichten Cholesaanfalle eine schwer $\mathrm{zu}$ beseitigende Intermittens eintrat, und umgekehrt in Fällen, wo der Anfall mit einem heftigen Schủttelfrost begann.

II. Zur pathologischen Anatomie.

Es ist bekannt, dass jene auffallende, für das Tastgefühl so unangenehme sogenannte Eiskälte der asphyctischen Kranken sich

*) Guttman und Baginsky, Centralblatt No.44, Stackwis, Centralblatt No. 54.

**) Charité-Annalen III. 
kurz nach dem Tode verliert, und einer normalen Temperatur Platz macht; weniger bekannt ist, dass auch bei den unmittelbar nach dem Tode sehr collabirten Leichen der Collapsus allmählich sich verringert, und je später man die Leiche wiedersieht, desto mehr vermisst man das eingefallene faltige verzerrte Gesicht, wie es sich uns am Krankenbette präsentirte. Bei Individuen von heller Hautfarbe, namentlich jungen Mädchen, haben wir am zweiten oder dritten Tage nach dem Tode die dunkle Röthe der Wangen wiederkehren sehen, wie sie, nach Aussage der Angehörigen in gesunden Tagen gewesen. Ebenso auffallend ist es, dass jener so fade penetrante Geruch, den alle Aspbyctische verbreiten, und ibre Nähe für längere Zeit unerträglich macht, unmittelbar nach dem Tode sich verliert. Alle diese Erscheinungen haben wir nur bei den im asphyctischen, niemals bei den im typhoiden Stadium Verstorbenen gesehen. Hingegen haben wir die Ejaculatio seminis ( $G$ üterbock) in asphyctischen wie typhoiden Fällen gefunden und mikroskopisch festgestellt, jedoch bei den im Typhoid Verstorbenen nur dann, wenn eine unerwartete Verschlimmerung der Krankheit plötzlich den Tod herbeigeführt. In einem Falle von protrahirter Asphyxie haben wir den Samenaustritt nicht gefunden, in anderen äbnlichen Fällen wurde leider nicht darauf geachtet. Hiernach scheint zur Ejaculation der rasche Eintritt des Todes nothwendig zu sein.

Den Schottin'schen Belag haben wir in den Epidemien der Jahre 1853 und 1855 sehr häufig, in der letzten Epidemie nur in einem Falle zu beobachten Gelegenheil gehabt.

Mit grossem Interesse baben wir in allen Epidemien die pathologischen Veränderungen des Darmkanals verfolgt, und aus nahezu 300 Sectionen die Ueberzeugung gewonnen, dass die Heftigkeit der Krankheitserscheinungen keinen Schluss zulasse auf einen erheblichen pathologischen Befund im Darmkanal; und umgekehrt.

Von 21 genau verzeichneten Fällen *) aus den drei letzten

*) Wir können versichern, dass etwa die doppelte Zahl von ähnlichen Fällen uns zu Gesichte gekommen, deren Sectionsbefund aber im Drange der Geschäfte nicht genau notirt worden ist. Wir wählen Fälle aus den verschiedenen Epidemien, um dem Einwurf zu begegnen, als wären die Er scheinungen blos Eigenthümlịchkeiten einer einzigen Epidemies. 
Epidemien lassen wir kurz einige mit Weglassung der unwesentlichen Momente folgen:

I. 1853 4. September. C. L., 36 Iahre alt, sehr Iräftiger Mann, Portier der Heilanstalt, aus Furcht vor Cholera mit Kranken nie in Berührung gekommen,. erkrankt um $10 \mathrm{Jhr}$ Morgens an Durcbfali. Die noch gelb gefärbten Stähle füllten ein mässig grosses Nachtgescbirr. Der zuletzt am 1 Uhr gelassene Urin $(50 \mathrm{Ccm}$.) war noch vorbanden und enthielt kein Eiweiss. Als das Erbrechen sich öfter wiederholte, requirirte er ärztliche Hälfe. Um 2 Uhr auf die Station gebracht, bot er im höchsten Grade alle Erscheinungen der Asphyxie dar, von denen wir rur hervorheben, dass seine Reiswasserstüble während seines Aufenthaltes auf der Station kaum ein Stechbecken füllten, und Erbrechen nur einmal stattfand. Er starb um $4 \frac{1}{2}$ Uhr Nachmittag trotz aller angewandten Mittel.

Section am 5. September 11 Uhr. Sämmtliche serösen Häute trocken, klebrig. Lungen an der Oberä̈che emphysematös, die unteren hinteren Lappen blutreich. An der Basis des Herzens grosse Ecchymosen, linker Ventrikel contrahirt, leer; rechter Ventrikel gefüllt mit weissen Blatgerinnseln. - Mesenterialdrüsen sehr geschwellt, Magen und Dünndarm mit Flüssigkeit ziemlich gefüllt, von blassrother Farbe. Die Sehleimhaut meist vom Epithel entblösst, capilläre Injection weit verbreitet. Solitäre Drüsen und Peyersche Plaques nur im anteren Theil des Jejunum sichtbar, von letzteren nur ein grösserer und drei kleinere rorhanden. Nieren im hyperämischen Stadium, Kapsel leicht abziehbar.

II. 1855 (Journal No. 201) 14. September. Marie Sasse, Amme, 26 Jahre alt, erkrankte in der Fischerstrasse 40 Nachts um 4 Ubr. Um $6 \frac{3}{4}$ Uhr wird sie im asphyctischen Zustande ins Lazareth gebracht and starb um \& Uhr Nachmittags. Aus der Krankengeschichte wollen wir nur erwähnen, dass die Mammae knollig angeschwollen und schmerzhaft blieben, auf Druck secernirten sie Milch von dickflüssiger Consistenz.

Section 15. September Morgens. Lebhafte allgemeine Hyperämie des Darmkanals, Schwellung der Schleimhaut in grosser Ausdebnung, starke Desquamation der Epithelien schon im oberen Theil des Jejunum. Hie und da geschwellte Brunner'sche und Solitärdrüsen, jedoch in mässiger Anzahl. Vier stark prominirende weissliche Peyer'sche Plaques, von denen die grösste zwei Zoll lang war, in der Nähe der Bauhin'schen Klappe. Mesenterialdrüsen hauptsächlich in dieser Gegend geschwellt, an anderen Stellen weniger.

Der Uterus mit blutigem Schleim gefüllt, die Yagina sebr hyperämisch.

III. 1866 20. September. Gottlieb Müller, Portier, Heidestrasse 33, 51 Jahre alt, in der Nacht an Durchfall erkrankt, wenig Erbrechen. Um $11 \frac{1}{4}$ Uhr Mittags wird er in stärkster Asphyxie eingeliefert. Reiswasserstïhle gehen unwillkürlich ab, etwa einige Fundert Ccm., Erbrechen nicht vorhanden. Tod am 21. September Morgens $5 \frac{1}{2}$ Uhr. Im Lazareth hat er $1200 \mathrm{Ccm}$. Selterwasser und $300 \mathrm{Ccm}$. Wasser getrunken.

Section am 21. September. Der Darmkanal ziemlich stark byperämisch, enthielt mehr als $2000 \mathrm{Ccm}$. weissflockiger Reiswasserflüssigkeit. Nur ein einziger 
$\frac{1}{2}$ Zoll Panger Peyer'scher Drüsenhaufen und wenig geschwellte Follikel an der Bauhin'schen Klappe waren bei der genauesten Untersuchung zu finden. Das Blut schwarz, theerartig aber nicht eingedickt.

Wir verweisen zum Ueberfluss auf einige von Reinhardt und Leubuscher als Anhang zum Berichte der 48er Epidemie gelieferten Sectionsbefunde (dieses Archiv Bd. II. Heft Ill.). Dort wird im XXsten „ziemlich heftigen Choleraanfall“ S. 575 angegeben: „Dünndärmschleimbaut mässig hyperämisch, die Peyer'schen Plaques und solitären Drüsen unbedeutend geschwollen." Im XXIsten "schweren Choleraanfall" wird S. 579 im Sectionsbefund mitgetheilt: "Nur im untersten Theil des Ileum geringe Anschwellung der solitären Drüsen. Die Peyer'schen Plaques nicht verändert."

Andererseits finden wir in leichten Cholerafällen, die später an Pneumonie oder im Puerperium verstorben sind, sehr schwere bereits zurïckgebildete Läsionen des Darmkanals, welche in keiner Weise den geringfügigen Erscheinungen der Cholera intra vitam entsprechen, so dass wir nicht berechtigt sind, aus dem intensiven und rapiden Auftreten der Choleraerscheinungen auf die In- und Extensität des pathologischen Prozesses im Darmkanal zu schliessen und umgekehrt.

Man hat überhaupt zu viel Gewicht auf die Schwellungen der Follikel in der Cholera gelegt. Ja man hat sogar aus dem Umstande, dass nach einem sechsstündigen Verlaufe der Cholera schon sehr ausgedehnte Infiltrationen der Follikel sich vorfinden, auf ein längeres Incubationsstadium der Cholera geschlossen, in welchem diese Schwellung sich allmählich vorbereitet haben soll. Abgesehen davon, dass ihre geringe oder grössere Infiltration, wie wir eben gesehen, in keinem Verhältniss zu den geringeren oder grösseren Erscheinungen intra vitam stehen, können sie unmöglich als · pathognomonisch für die Cholera betrachtet werden, da sie ja im Typhus nie fehlen und im Scharlach nicht selten gefunden werden. Ja es ist mehr als wahrscheinlich, dass jene Schwellungen der solitären und Peyer'schen Drüsen als eine physiologische Veränderung während der Verdauung zu betrachten ist. Schon im Jahre 1834 hat Böhm in seiner vortrefflichen Dissertation*) Abbildungen von Peyer'schen Plaques geliefert,

*) De Glandularum intestinalium structura penitiori. 
welche dem Darme eines plötzlich am Schlagfluss verstórbenen bis dahin gesunden Mannes entnommen sind. Ein Unterschied zwischen jenem Befunde und den geschwollenen Plaques, die man in der Cholera sieht, ist schwerlich aufzufinden. Mit Recht sagt daher Böhm in seiner klassischen noch jetzt maassgebenden Schrift ${ }^{*}$ ) p. 71: "Der jedesmal von dem Verdauungszustande bedingte, ganz zufällige Grad der Füllung oder die Leere der Kapseln ist es, woron es lediglich abhängt, ob man die solitären und Peyer'schen Drüsen überhaupt finden kann, ob sie ein anderes Mal leicht deutlich sind, oder ob sie sogar ein überraschendes Ansehen gewinnen, ohne dass in dem einen oder anderen Falle die geringste krankhafte Veränderung im Spiele zu sein brauche. Man hört häufig den Schluss, Peyer'sche Drüsen seien krankhaft angeschwollen, wo im Gegentheil die strotzende Füllung der Kapseln nur einen Beweis ihrer ungetrübten kräftigen Function abgaben." In dieser Voraussetzung haben Thiersch und Buhl**) einen Verbrecher vor seiner Hinrichtung passend genährt, und nach dem Schwertstreiche das Verhalten seines Darms und Mesenterialdrüsen analog der Cholera gefunden. Es scheinen demnach jene Schwellungen der Darmdrüsen nur auf eine plötzliche Unterbrechung des physiologischen Verdauungsactes hinzudeuten und daher kommt es, dass sie in Cholerafällen meist da sich spärlich vorfinden, wo längere Zeit Dyspepsien und Diarrhöen vorangegangen, die eine karge Diät erforderten.

Mit diesen Schwellungen der Drüsen geht meist schon nach 24-48 Stunden eine Veränderung vor, so dass sie jenes bekannte siebartige, reticulirte Ansehen gewinuen. Mit Unrecht wird diess von einigen Autoren als ein Rückbildungsprozess angesehen. Schon Virchow ${ }^{* *}$ ) hat die pathologische Wichtigkeit bestritten und den ganzen Vorgang als ein Leichenphänomen betrachtet. Da sich aber die reticulirten Plaques bei Sectionen, die kurz nach dem Tode gemacht sind, auch vorfinden, so müssen wir sie füglich in die Kategorie des allgemeinen Zerstörungsprozesses, wie

*) Die kranke Darmschleimhaut in der Cholera (Berlin, 1838).

**) Zeitschrift für rationelle Medicin Bd. VI. Hft. I. S. 62.

***) Medicinische Reform No. 10. 
er auf der Darmschleimhaut an allen Gebilden sich manifestirt, bringen. Durch die massenhafte Transsudation von Flüssigkeit durch die Darmschleimhaut wird ihr Epithel streckenweise locker, ihre Zotten aufgequollen, abgelöst und förmlich macerirt. Dieser Macerationsprozess ergreift dann auch die drüsigen Gebilde, zerstört die Oberfläche der gefüllten Kapseln, welche ihren Inhalt entleeren und jenes siebförmige Ansehen gewinnen, um endlich selbst auszufallen und eine Tabula rasa zurückzulassen. Rückbildungsprozesse im eigentlichen Sinne kann man höchstens an den solitären Follikeln erkennen, die sich durch Resorption verkleinern, die Plaques hingegen exfoliiren meist vollständig. Die Stelle, wo sie gesessen, ist vertieft, durchsichtig, fast gefässlos.

Bei den in der eigentlichen Asphyxie Verstorbenen baben wir in keiner der drei Epidemien Diphtheritis beobachtet. Selbst in der letzten Epidemie, wo wir intra vitam Diphtheritis der Scheide und auf den Tonsillen sehr häufig gesehen haben, sind im Darmkanal nur bei den im Uebergangsstadium und im Typhoid Verstorbenen Diphtheritis gefunden worden. Einmal fanden wir zwei tiefe diphtheritische Geschwüre in der Gallenblase. Am ausgedehntesten waren die diphtheritischen Exsudate, nach dem Stadium der Krankheit mit oder ohne Geschwürsbildung, am Rectum in der Nähe des Anus, wo sie förmlich einen Kranz bildeten. Ebenso zahlreich waren sie in der Vagina anzutreffen, in einem Falle waren in exquisiter Weise erbsengrosse, weit hervorragende, weissgraue, harte Infiltrationen sichtbar vom Muttermunde bis zum Ausgang der Scheide, die tief bis ins submucöse Bindegewebe reichten.

Bämorrhagische Infarcte waren im Jahre 1853 und 1855 in der Milz, Leber und Nieren selbst im asphyctischen Stadium sehr häufig anzutreffen, in der letzten Epidemie auffallender Weise selbst nicht einmal im Typhoid.

Hr. Dr. Cohnheim, der die Güte hatte, öfter in unserem Lazareth Sectionen zu machen, machte uns auf die Anschwellungen der Papillae circumvallatae an der Zungenwurzel aufmerksam, die wir dann constant in allen Fällen gefunden haben. Diese Anschwellungen haben wir selbst noch bei den im Typhoid Verstorbenen gefunden und fehlten auch nicht, natürlich in viel kleinerem Maasse, bei den im Uterus verstorbenen Frühgeburten (s. u.). 
Der Befund an der Niere isi je nach der Dauer der Krankbeit verschieden. In rapid verlaufenen Fällen erscheinen die anatomischen Veränderungen ganz analog denen der Stauungshyperämie. Die Niere ist noch von normaler Grösse, die Kapsel leicht adhärent, dunkelbläuliche baumförmige Injectionen an der Oberfläche, die Corticalsubstanz noch dunkelroth, ebenso die Pyramiden an der Basis, während der den Papillen zunächst gelegene Theil schon eine weissliche Farbe und derbe Consistenz annimmt, die Glomeruli sind intakt, die venösen Gefässe erweitert. Bei längerer Dauer der Asphyxie, zuweilen schon nach 24 Stunden fanden wir die Corticalsubstanz graugelb enträrbt und ebenso die Degeneration der Pyramiden nach der Basis zu vorgeschritten. Im Typhoid endlich erschien die Corticalis bedeutend vergrössert und keilförmig zwischen die Pyramiden gedrängt, schlaff, leicht zerreisslich, intensiv blassgelb gefärbt, die Pyramiden, namentlich die in der Mitte der Niere gelegenen, total fettig degenerirt. Selten fanden wir die Nieren im atrophischen Stadium.

Am Herzen fehlten die kleinen Ecchymosen an der hinteren Fläche der Basis selten, sie waren sogar noch bei den im $\mathrm{Ty}$ phoid Verstorbenen anzutreffen, wenn auch schon etwas erblasst.

Das Lungengewebe war auffallend trocken, vorn blass, hinten und namentlich unten blutreicher, zuweilen ödematös; hypostatische Pneumonie fand sich zuweilen schon bei den in der protrahirten Asphyxie Verstorbenen.

Auf die einzelnen Befunde im Typhoid kommen wir später noch zurück, sowie auf die Befunde bei den fötalen Leichen.

\section{Nosologisches.}

Der Choleraprocess charakterisirt sich durch zwei Reihen von Erscheinungen, welche in Bezug auf ihre pathognomonische Wichtigkeit wesentlich von einander verschieden sind: eine positive und eine negative Reihe von Symptomen. Die positiven Symptome sind hauptsächlich: profuse ungefärbte Ausleerungen, Erbrechen, Krämpfe, Cyanose; die negativen: Anurie, Aphonie, Pulslosigkeit. Die erste Symptomengruppe gehört eigentlich der sogenannten Cholerine an (aus welcher bekanntlich sich nicht. immer und nothwendig die Cholera entwickelt), ja sie kann sogar bis auf die Cyanose in der ausgebildeten Cholera, dem sogenann- 
ten asphyctischen oder paralytischen Stadium derselben gar nicht zur Erscheinung kommen; hingegen ist die negative Symptomenreibe pathognomonisch für die eigentliche Cholera asphyctica.

Man ist mit Recht zu allen Zeiten bemüht gewesen, eine bestimmte Grenze zwischen Cholerine und Cholera zu ziehen. Man hat Anfangs die Reiswasserstühle als charakteristisch für die Cholera angesehen, aber man hat sich sehr bald überzeugt, dass in vielen asphyctischen Fällen die Farbe der Stühle gallig erscheint (wie wir sie aus allen drei Epidemien in nicht geringer Zahl aufzuweisen haben), andererseits aber schon bei einfachen verbreiteten Intestinal-Katarrhen mit Affection der Schleimhaut des Ductus choledochus, die schönsten Reiswasserstühle auftreten. Wir haben erst in jüngster Zeit, nach dem Erlöschen der Epidemie, ein Kind an Masern behandelt, welches bei vollkommen normalem Verlaufe der Krankheit, 24 Stunden lang, weissflockige Stühle entleerte. Alle übrigen Symptome sind inconstant und kommen auch bei anderen Krankheiten vor. Der sicherste Grenzstein ist und bleibt die Anurie; mit dem Cessiren der Urinsecretion beginnt das sogenannte Stadium algidum und das Wiedererscheinen des Urins beschliesst dasselbe. Wir kommen später auf die Anurie ausfübrlich zurück und wollen jetzt obenerwähnte hauptsächlichen Choleraerscheinungen der Reibe nach betrachten, wie sie sich uns während drei Epidemien im Lazareth darstellten.

I. Dejectionen. Zu allen Zeiten und am meisten in der letzten Zeit hat man das grösste Gewieht auf die Transsudationen des Darmkanals gelegt. Man hat alle anderen Vorgänge zurückgeführt auf die durch die Dejectionen herbeigeführte Eindickung des Blutes. Wir werden sehen, in wieweit diese scheinbar klare Betrachtungsweise der Wirklichkeit entspricht.

Die Beschaffenheit der Dejectionen hat $B o ̈ h m$ in seiner vortrefflichen Schrift*) am schärfsten geschildert. Sie bestehen aus einer schwach gelblich weissen Flüssigkeit, aus welcher sich beim Stehen ein bedeutender Bodensatz von gelblich weissen Flocken scheidet. In dieser schleimigen Masse haben wir, mit Böhm, kein Cylinderepithel mehr finden können. Die abgestossenen Darmzotten sammt dem zahlreichen Epithel werden schon im Darmkanal so 
macerirt, dass nur ihre Trümmer in den Dejectionen sichtbar sind. Jene grösseren darin schwimmenden weissen Flocken bestehen aus einer gallertartigen Masse, die vielleicht als ein Secret der Darmdrüsen zu betrachten sein dürfte.

Die Dejectionen reagiren selten neutral, meist alkalisch yom Ammoniak-Corbonat, das aus dem ausgeschiedenen Harnstoff sich rasch bildet. Das specif. Gewicht schwankt zwisehen 1006-1013.

Die Quantität der Dejectionen variirt bedeutend je nach dem Charakter der Epidemie und der Häufigkeit der sogenannten enterischen oder besser transsudativen Fälle. Während man in den echt asphyctischen oder paralytischen Fällen während ihres ganzen Aufenthaltes im Lazareth kaum 500-1000 Ccm. Dejectionen erhält, siebt man in transsudativen Fällen neben Tausenden Ccm. Erbrochenen noch bis $5000 \mathrm{Ccm}$. Dejectionen in 24 Stunden abgehen. So gehörten in den Epidemien der Jahre 1853 und 1855 die transsudativen Fälle zu den Seltenheiten. Die Stühle waren meist von dickschleimiger Beschaffenheit, der wässerige Theil der Dejectionen sehr spärlich vertreten.

Wir verweisen hier auf die der nachstehenden Arbeit unseres Assistenten Hrn. Bruberger angefügten Tabellen, welche übersichtlich das Verhältniss zwischen Einnahme und Ausgabe von Flưsssigkeiten in der Cholera darstellen. Wir werden später noch öfter auf diese Tabellen Bezug nehmen müssen, wollen daher jetzt nur hervorheben, dass der Patient der Tab. I. in seiner Asphyxie $6662 \mathrm{Ccm}$. Flüssigkeit mehr transsudirt als eingenommen und trotzdem seine Asphyxie schon am anderen Morgen mit grosser Leichtigkeit überwunden hat, ohne auch nur die geringste typhoide Anfechtung zu haben. Die Patientin der Tab. II. hat in 24 Stunden sogar $12300 \mathrm{Ccm}$. mehr verausgabt als eingenommen ohne auch nur in die Asphyxie überzugehen. Andererseits haben die beiden in Tab. III. aufgeführten Pat. inclus. der bei der Section gefundenen Darmflüssigkeit nur $2130 \mathrm{Ccm}$. und noch viel weniger transsudirt und sind in der Asphyxie in kurzer Zeit gestorben. Aus diesen wie aus den noch später anzuführenden Fällen, welche nur als Paradigmata für eine grosse Reihe von Füllen zu betrachten sind, geht hervor, dass die massenhaften Transsudationen in der Cholera nicht so schädlich für den $0 \mathrm{r}$ ganismus sind, wie man jetzt allgemein anzunehmen pflegt. Wir 
werden noch später sehen, dass bei geringen Transsudationen das Typhoid auch' ein viel schwereres wird.

II. Erbrechen. Das stossweise, bei offenem zugespitzten Munde, in einem bogenförmigen Strahl, zur eigenen Ueberraschung des Patienten eintretende Erbrechen, bezeichnet nächst der Anurie am sichersten den Beginn des Cholera-Anfalls. Gewöhnlich pflegt mit dem Auftreten desselben auch die Urinsecretion zu cessiren, zuweilen etwas später. Das Erbrechen fehlt in den seltensten Fällen ganz, hört aber constant auf der Höhe der Krankheit auf. Daher ist das Aufhören des Erbrechens ohne Nachlass der Allgemein-Erscheinungen von prognostischer Wichtigkeit.

Der Akt des Erbrechens selbst wird nicht, wie Hamernik behauptet, durch die Bauchpresse und den hierdurch auf den Magen bewirkten Druck eingeleitet, sondern durch die antiperistaltischen Contractionen 'des Magens selbst, wobei natürlich wie bei jedem Brechakt die Bauchmuskeln mitthätig sind. Dass die Aktion der letzteren erst secundär eintritt, kann man sich überzeugen, wenn man eine Zeit lang die Hand auf der Magengegend liegen lässt. Beim weiteren Fortschreiten der Krankheit hört das Erbrechen auf, obgleich die Bauchpresse noch thätig ist. Vergebens bemüht sich zuweilen der Pt., durch tiefes Hineinstecken des Fingers in den Pharynx den Brechakt wieder heryorzurufen, es gelingt ihm so wenig wie den Aerzten durch Emetica. Die antiperistaltischen Contractionen des Magens haben dann wie die peristaltischen des Dünndarms aufgehört. Die Darmflüssigkeiten fliessen nach dem Gesetze der Schwere bei jeder Bewegung des Körpers unten unfreiwillig ab. -

Das Erbrechen besteht aus einer klaren gelblich weissen Flüssigkeit mit einem schleimigen Bodensatz, welcher mikroskopisch noch lange Zeit Speisereste erkennen lässt, Epithelialgebilde aus Rachen und Oesophagus, Buccalepithel und viel Schleimkörperchen. Vibrionen sind von uns nicht selten darin gefunden worden. Die Flüssigkeit reagirt in frischen Fällen noch sauer, meist neutral und selten alkalisch.

Die Quantität des Erbrochenen hängt allerdings von der Menge der eingenommenen Flüssigkeit $a b$, besteht aber keineswegs nur aus dieser, wie Buhl fälsehlich behauptet, sondern übersteigt diese oft sehr bedeutend. Hierfür spricht schon das 
einfache Verhältniss der eingenommenen Flüssigkeit zum Erbrochenen, wie es in einer Reihe von Fällen in unserem Lazareth festgestellt ist. So hat z. B. in einem frisch eingelieferten Falle die Menge des Erbrochenen $21250 \mathrm{Ccm}$. in 24 Stunden betragen, während in derselben Zeit nur $9000 \mathrm{Ccm}$. Selter- und $1200 \mathrm{Ccm}$. Eiswasser getrunken waren. Es ist also in diesem Falle $12300 \mathrm{Ccm}$. Flüssigkeit mehr ausgebrochen als getrunken, also mehr als die Hälfe des Erbrochenen ist aus dem Blute und der anderen Körperflüssigkeit entnommen.

Blut haben wir im Erbrochenen in der Epidemie des Jahres 1853 in 2 Fällen, im Jahre 1855 in vier Fällen gesehen, von denen in 2 Fällen die Quelle der Blutung bei der Section bat nachgewiesen werden können. In dem einen Falle war ein altes Ulcus ventriculi, in dem anderen Carcinom vorhanden. In der letzten Epidemie ist kein blutiges Erbrechen beobachtet worden. -

Galliges Erbrechen haben wir in allen drei Epidemien selten in der Asphyxie, oft im Uebergange zum reactiven Stadium beobachtet, jedoch stets mit Ausgang in Genesung.

III. Die Muskelkrämpfe sind in keiner der drei Epidemien constant vorhanden gewesen, vielmehr bäufig vermisst worden. Bei sehr kräftigen Individuen sind sie häufiger und schmerzhafter als bei schwächlichen Personen, bei Männern häufiger als bei Frauen. Sie sind nicht blos an den Waden, sondern sehr häufig auch an den oberen Extremitäten beobachtet worden. In einem sehr rapid verlaufenen Falle der letzten Epidemie waren die Krämpfe auf fast sämmtliche Muskeln des Körpers verbreitet, namentlich auf Rücken- und Nackenmuskeln, so dass die Patientin bewegungslos in den heftigsten Schmerzen (trotz Morphium-Injektionen) dalag. Dass sie keine Folge der Transsudation sind geht schon aus dem Umstande hervor, dass sie auf der Höhe der Krankheit verschwinden und nur in den allerseltensten Fällen bis an's Ende andauern. Der Krampf des Zwerchfells (Singultus) tritt erst nach der Asphyxie ein.

IV. Auch die Cyanose ist kein charakteristisches Symptom des Stad. algidum. Man sieht sie meist schon bei noch fühlbarem Pulse, namentlich an dem reichen Capillargefässnetze der Lippen, der Zunge, der Glans penis. Aber die Cyanose ist bereits ein Uebergangssymptom zum Stad. algidum und wo sie vorhanden 
war, da ist die Asphyxie nicht ausgeblieben. Sie verliert sich in der Reaction und an der Leiche nur allmählich, die tief blaue Färbung der Glans penis aber scheint post mortem gar nicht mebr zu schwinden. -

Wir kommen nunmehr zur negativen Reihe der CholeraSymptome, die constant niemals im Stadium algidum fehlen und in keiner anderen Krankheit in dem Grade vorkommen.

I. Anurie. Noch immer gibt es Aerzte, sogar solche, die die Cholera-Literatur bereichern, welche die Thatsache, dass in der Asphyxie kein Tropfen Urin gelassen wird, anzweifeln. Ihr Irrthum beruht darauf, dass man in der Asphyxie meist mit dem Katheter eine kleine Quantität Urin entleeren kann, der aber keineswegs während der Asphyxie in die Blase gelangt ist, sondern schon vorher secernirt, durch eine spastische Contraction des Sphincter zurückgehalten wird. Diese spastische Contraction ist in der Asphyxie constant und erschwert bekanntlich das Katheterisiren in hohem Grade. Dieser letzte vor der Asphyxie gelassene Urin unterscheidet sich wesentlich von dem nach Ablauf des Stad, algidum secernirten sogenannten ersten Urin. Jener ist stets von hellerer Farbe, meist ohne Eiweiss, zuweilen eiweisshaltig, niemals aber enthält er Faserstoffcylinder. Am deutlichsten tritt der Contrast hervor in den Fällen, wo die Asphyxie aus den Prodromalstadien sich rapid entwickelt hat, hier ist der letzte Urin von normaler Beschaffenheit ohne Eiweiss, ohne Cylinder, nur etwas concentrirter als im normalen Zustande. Wo die Asphyxie aber sich allmählich entwickelt, da wird in der Uebergangszeit zum Stad. algidum ein eiweisshaltiger Urin entleert, aber ebenfalls ohne Cylinder. In den Epidemien von 1853 und 1855 gehörte der Eiweissgehalt des letzten Urins zu den allergrössten Seltenheiten, häufiger wurde Eiweiss in der letzten Epidemie gefunden. Wir wissen bereits, dass in den ersten Epidemien viel mehr rapid verlaufene Fälle im Lazareth waren, als in der letzten Epidemie, daher der Unterschied in dem Befunde. -

Dass während der Asphyxie kein Tropfen Urin secernirt wird, davon kann man sich zum Ueberflusse dadurch überzeugen, dass man jeden in der Asphyxie eingelieferten Patienten, wie wir es gemacht haben, sofort katheterisirt, den Katheter in der Blase um- 
dreht und einen Druck auf die Blasengegend ausübt, um jeden Inhalt selbst vom Grund der Blase zu entleeren und man kann sicher sein, während der ganzen Dauer der Asphyxie keinen Tropfen in der Blase mehr zu finden, so oft man auch katheterisiren mag. -

Das Wiedereintreten der Urinsecretion beschliesst daher die Asphyxie, nicht die spontane Entleerung des Urins, da diese wegen der fortdauernden spastischen Contraction des Sphincter erst später einzutreten pflegt und es uns nur auf die restituirte Function der Niere, nicht der Blase ankommt. Wir haben daher zur gelegenen Zeit, auf die wir später zurückkommen, katheterisirt, bis wir den sogenannten ersten Urin entleert haben, den man bei einiger Uebung sofort als solchen erkennt, und von dem folgenden zweiten und dritten Urin mit Sicherheit unterscheidet. Der erste Urin ist trübe, charakteristisch braunroth, mit Flocken und Fetzen vermischt. Die später secernirten Portionen werden immer klarer, durchsichtiger, verlieren das braunrothe Ansehen, werden saturirt gelb, und wenn dann in der Reconvalescenz die drei- und vierfache Menge des normalen Urins gelassen wird, wird er so blassgelb, dass er ganz das Aussehen eines diabetischen Harnes annimmt. Tritt keine Reconvalescenz, sondern Uebergang ins Typhoid ein, so behält der Urin eine trübe braungelbe Farbe, die sich aber doch von dem erstsecernirten leicht unterscheidet. - Mikroskopisch sieht man in ihm Plattenepithel aus dem ganzen uropoetischen Apparat, meist von der Blasenschleimhaut, viele hyaline oder fettig entartete FibrinCylinder von dem verschiedensten Kaliber.

Die Menge der in den ersten Urinen vorhandenen Cylinder ist von grosser Wichtigkeit auf den weiteren Verlauf der Urinsecretion und somit der Krankheit selbst. Nach unserer Beobachtung werden die Cylinder in den zur Genesung führenden Fällen schon in den ersten Urinen in ungeheurer Menge ausgestossen, verlieren sich dann allmählich und yerschwinden vollständig, sobald die Urinsecretion ihr Maximum erreicht hat. Hierhin gehören auch die in Tab. I. u. II. aufgeführten Fälle. Bei zu lange andauernder Anurie hingegen erscheinen die Cylinder im ersten Urin sehr sparsam, auch in den folgenden in geringer Anzahl und fettig degenerirt. Dieser sparsamen Ausstossung von Cylindern entspricht aucb die folgende spärliche Urinsecretion. Hierzu gehören die unten in Tab. IV. u. V. 
aufgefïhrten Todesfälle. Je reichlicher demnach die Ausstossung der Cylinder stattfindet, desto günstiger ist der Verlauf der Krankheit. Mit der Vermehrung der Urinsecretion vermindern sich die morphologischen Elemente desselben, bis er das normale Verhalten erreicht. Auch Vibrionen sind selbst in frisch gelassenem, nicht alkalischem Urin öfter beobachtet worden.

Die Quantität schwankte am 1sten Tage zwischen 100-500 Ccm., stieg dann in normal verlaufenen, d. h. zur Reconvalescenz führenden Fällen stetig, bis sie am 5ten oder 6ten Tage das Maximum oft $\mathrm{zwischen} 4-5000 \mathrm{Ccm}$. erreicht hatte, und sank dann allmählich wieder bis zur Norm. In Fällen, die zum schweren Typhoid (Urämie) führen, bleibt die Urinsecretion eine beschränkte (200-1000 Ccm.).

Wir verweisen hier wieder auf die am Schlusse des Heftes befindliche Tabelle IV. u. V., aus denen durch Vergleichung mit den anderen Tabellen der Contrast in der Urinsecretion deutlich hervortritt. Während der zur Genesung führende Fall der Tabelle I. am 4ten Tage bereits $2000 \mathrm{Ccm}$. entleert, hat der in Tabelle IV. mit schwerem Typhoid behaftete nur $630 \mathrm{Ccm}$. aufzuweisen. Bei der in der Reconvalescenz begriffenen Patientin der Tabelle II. ist am 6ten Tage $2475 \mathrm{Ccm}$. Urin notirt, während der an Urämie verstorbene Pat. der Tab. V. am 6ten Tage nur 900; Ccm. Urin gelassen hat.

Die sehr stark saure Reaction des ersten Urins wird mit der zunehmenden Quantität allmählich schwächer und kann selbst neutral werden. Alkalische Reaction haben wir nur bei begleitendem Blasenkatarrh gefunden. -

Das specif. Gewicht schwankt zwischen 1012-1024, später sinkt es, dem zunehmenden Wassergehalt entsprechend, in maximo auf 1004-1008. -

Der erste Urin nach der Asphyxie enthält stets Eiweiss in erheblicher Menge. Diejenigen Autoren, welche den ersten Urin eiweisslos gefunden, haben sicherlich ihn mit dem oben gesehilderten vor der Asphyxie secernirten letzten Urin verwechselt. Wir haben nunmehr während drei grösseren Epidemien mit grosser Genauigkeit die Urinsecretion beobachtet, ohne auch nur ein einziges Mal den ersten. Urin eiweisslos gefunden $\mathrm{zu}$ haben. Schon nach einer 5-6stündigen Stockung der Urin- 
secretion, haben wir den wiedereintretenden Urin eiweisshaltig gefunden mit Faserstoff-Cylindern. Wir können daher den Eiweissgehalt des Urins hinterher zur Feststellung der Diagnose, ob Cholera oder Cholerine vorangegangen, mit vollem Rechte benutzen. - Auch die späteren Urine entbalten Eiweiss, jedoch vermindert sich der Eiweissgehalt allwählich von der Flockenbildung zur Opalescenz und schwindet vollständig, noch ehe die Urinsecretion die höchste Quantität erreicht hat. Die Dauer und die Stärke des Eiweissgehaltes der späteren Urine hängt $a b$ von der Dauer der vorangegangenen Anurie. Je länger die Stockung der Urinsecretion angedauert, desto länger und intensiver ist der Eiweissgehalt in den späteren Urinen. -

Wir sind im Lazarethe bäufig in der Lage gewesen, bei Kranken, die in einem der Cholera eigenthümlichen apathischen oder somnolenten Zustande uns eingeliefert worden, das Stadium der Krankheit lediglich aus dem Befunde in der Blase festzustellen, so dass wir die Beschaffenheit des Urins, sowie das Ausbleiben desselben als das wichtigste diagnostische und prognostische Hülfsmittel in der Cholera betrachten müssen. Auf die Prognose kommen wir noch zurück und wollen jetzt noch des $\mathrm{Zu}$ sammenhanges willen die chemische Beschaffenheit des Urins weiter verfolgen.

Die Harnstoffausscheidung in den ersten Urinen ist eine abnorm geringe. Sie betrug im ersten Urin im Mittel von 30 Analysen 11 p. M., steigt in Fällen, die zur Genesung führen sehr rasch, erreicht aber das Normale von 23 p. M. nur selten, bleibt vielmehr auf $16-20$ p. M. stehen. Die absolute Quantität von Harnstoff wird in Genesungsfällen immer bedeutend vermehrt, und es werden dann, wenn die Quantität des Urins auf ihr Maximum gestiegen ist, oft 70-80 Grm. in 24 Stunden ausgeschieden, so dass der Organismus das in früheren Tagen Versäumte wieder nachzuholen scheint, und die während der Asphyxie im Körper angesammelten Excretionsprodukte (Kreatinin, Harnstoff), von denen nur ein kleiner Theil durch die Transsudation ausgeschieden ist, nunmehr zu eliminiren sucht. In Făllen, wo die Genesung sich längere Zeit hinzieht, oder die in's Typhoid übergehen, steigt der relative Harnstoffgehalt auch um Weniges, der absolute dagegen bleibt entsprechend der geringen Quantität des Urins ein abnorm 
niedriger. Wir verweisen auf die in der Tabelle I. und II. verzeichneten Genesungsfälle im Vergleiche zu den im Typhoid Verstorbenen der Tabelle IV. und V., und die darin verzeichneten Werthe des ausgeschiedenen Harnstoffs, wollen aber noch ausserdem nachstehende drei Krankengeschichten der verschiedensten, Kategorie mittheilen, welche die Wichtigkeit der Harnstoftausscheidung begründen mögen. In dem ersten Genesungsfalle stieg die Harnstoffausscheidung auf $67,5 \mathrm{Grm}$. p. d. nach 24stündiger Anurie. Im zweiten Falle war nach 68stündiger Anurie die höchste Tagesausscheidung von Harnstoff $=19$ Gramme. Im dritten Falle hatte die Urinsecretion 76 Stunden cessirt, und die grösste Harnstoffausscheidung war nur 7,8 Gramme p. d.

1.

Leichter Cholerafall. Reichliche Transsudationen in den Darmkanal. 24stündige Dauer der Anurie. Reichliche Harnstoffausscheidung. Kein Typhoid. Genesung.

Friedrich Meerkatz, 24 Jabre alt, erkrankte am 12. August in den Vormittagsstunden mit Diarrhoe, die sich in kurzen Intervallen wiederholte, er wurde an selbigem Tage Abends 8 Uhr ins Lazareth aufgenommen und sagte aus, dass er ausserhalb nicht gebrochen und Urin noch im Laufe des Nachmittags gelassen habe. Er war von gutem Aussehen ohne Cyanose, seine Sprache sonor und voll, Temperatur etwas erhöht, Puls etwas frequenter als normal, beide Herztöne laut hörbar, Zunge belegt, Leib nicht schmerzhaft. Der Stuhlgang, der im Laufe des Abends gelassen wurde, war reiswasserähnlich. Urin konnte er, dazu aufgefordert, nicht entleeren. Injection von Chinin. muriat. Gr. viij.

13. August. Patient hat geschlafen, sein Puls ist klein und frequent, Temperatur gesunken, Erbrechen bat sich in der Nacht eingestellt, Patient entleert obne Anstrengung und Würgen grosse Massen Flüssigkeit, er trinkt viel, der willkürlich gelassene Stuhlgang exquisit reiswasserähnlich, es sind grosse Mengen. Katheterisation ergab früh leere Blase, Nachmittags 5 Uhr dagegen $5 \mathrm{Ccm}$. trüben Urins, von mässigem Eiweissgehalt, in denen viele hyaline Cylinder und Epithelialgebilde enthalten sind. Die Anurie hatte also etwa 24 Stunden gedanert. Im Laufe des Tages bessert sich sein Zustand etwas.

14. August. Patient hat geschlafen. Puls und Temperatur fast normal, Stühle noch sehr häufig aber schon gallig gefärbt, Erbrechen hat nachgelassen. Zunge sehr belegt, injicirte Conjunctiva. Urin noch nicht gelassen, die Katheterisation ergibt am frühen Morgen $15 \mathrm{Ccm}$. Urins von denselben Eigenschaften, wie die geringe Menge am vorhergehenden Tage. Um 2 Uhr Nachmittag lässt Patient spontan $145 \mathrm{Ccm}$. Urin, Reaction sauer, spec. Gew. 1,018, mässiger Eiweissgehalt, Cylinder, Epithelien, wenig Gallenfarbstoff, NaCl-gehalt $=0,5$ pr. mll. Harnst offgehalt, (nach Ausfällung des Eiweisses) 16 pr. mll. $=2,32$ Gr. 


\section{6}

15 August. Patient hat gut geschlafen, Puls und Temperatur fast normal, Erbrechen hat ganz aufgehört, Stuhl häufig, beginnt breiig zu werden. Heftiger Singultus. Urin spontan entleert. Es sind seit der gestrigen spontanen Entleerung 715 Ccm., sauer, spec. Gew. 1,015, Eiweissgehalt mässig, kieine Gallenfarbstoffreaction. Chlornatr. $=0,4 \mathrm{p} . \mathrm{m} .=0,5 \mathrm{Gr}$. HarnstoffgehaIt 17 pr. mll. $=12,155 \mathrm{Gr}$. Cylinder, Epithelien.

16. August. Patient hat gut geschlafen. Puls und Temperatur normal. Stïhle dünner wie gestern, Zunge noch stark belegt; Allgemeinbefinden zufrieden. Urin (von 24 Stunden) $1002 \mathrm{Ccm}$., sauer, spec. Gew. 1,011, Eiweissgehalt nur noch als 0palescenz vorhanden. Chlornatr. $=0,5$ p.m. $=0,5$ Gr. Gehalt an Harn stoff $=18,5$ pr. mll. $=18,537$ Gr. Cylinder, Epithelien, Eiterkörperchen, Spermatozoen.

17. August. Puls und Temperatur normal. Stühle noch dünn. Zunge belegt. Leichter Icterus. Allgemeinbefinden gut. Urin $3105 \mathrm{Ccm}$, sauer, spec. Gew. 1,009, Eiweiss verschwunden, keine Cylinder, wenige vereinzelte Epithelien. Chlornatr. $=0,7$ p.m. $=2,2$ Gr. Harnstoffgebalt 16,5 pr. mll. $=49,7 \mathrm{Gr}$.

18. August. Stühle noch dünn, sonst Wohlbefinden, Znnge reinigt sich. Urin $4500 \mathrm{Ccm}$., schwach sauer, spec. Gew. 1,007, kein Eiweiss, keine morphol. Elemente. Chlornatr. $=1,3$ p. m. $=5,8 \mathrm{Gr}$. Harnstoffgehalt $=15$ pr. mll. $=67,5 \mathrm{Gr}$.

19. August. Patient ist in voller Besserung. Stuhl breiig. Urin $4700 \mathrm{Ccm}$., schwach sauër, spec. Gew. 1,005. Chlornatr. $=0,8$ p. m. $=3,8$ Gr. Harnstoffgehalt $=13,5$ pr. mll. $=63,45 \mathrm{Gr}$.

20. August. Patient verlässt das Bett, einmaliger Stuhlgang. Urin $4400 \mathrm{Ccm}$., schwach sauer, spec. Gew. 1,005. Chlornatr. $=0,8$ p. m. $=3,5$ Gr. Harnstoffgehalt $=14$ pr. mll. $=61,6 \mathrm{Gr}$.

21. August. Stat. idem. Urin $3900 \mathrm{Ccm}$., schwach sauer, spec. Gew. 1,006. Chlornatr. $=0,8$ p.m. $=2, \breve{a}$. Harnst off́gehalt $=14,5$ pr. mll. $=56,5$ Gr.

22. August. Stat. idem. Urin 3350 Ccm., sauer, spec. Gew. 1,008. Harnstoffgehalt $=16$ pr. mll. $=53,6$.

23. August. Stat. idem. Urin $2300 \mathrm{Ccm}$., sauer, spec. Gew. 1,009. Harnstoffgehalt $=19$ pr. mll. $=43,7$ Gr. Geheilt entlassen.

2.

Schwere Asphyxie. Geringe Transsudationen. Anurie von etwa 68 stündiger Dauer. Cylinder werden in nur geringer Anzahl ausgestossen. Harnstoffausscheidung ungenügend. Schweres

Typhoid. Tod.

Wilhelm Heinrich, herrschaftlicher Diener, 27 Jahre alt, wurde aufgenommen am 25. September 1866 früh 6 Uhr.

25. September. Patient gibt an, dass er gestern Morgen, vielleicht in Folge einer Erkältung, plötzlich Durchfälle bekommen; am Abend mehrten sich dieselben und zugleich begann er zu erbrechen und za collabiren. Urin will er seit 12 
Uhr in der Nacht nicht entleert haben; er kommt von Güstrin und hat den Feldzug als Landwehrmann mitgemacht.

Stat. praes.: Pulslosigkeit, ganz gesunkene Temperatur. 0ppres. pector. Bedentende Cyanose, tiefliegende Augen, heftige Krämpfe. Vox choler., sehr belegte Zunge, mässiges Erbrechen und Reiswasserstühle in geringer Quantität. Inject. von Chinin muriatic. Gr. viij, kalte Einwickelung. Später Inject. von Strychnin. sulphic. $\frac{1}{3} \mathrm{Gr}$.

26. Sept. Patient bat wenig geschlafen. Puls nicht fühlbar, Temperatur gesunken, Erbrechen fast cessirt, Zunge reinigt sich. Herzstoss deutlich sichtbar. Stühle schon gallig tingirt. Der Katheterismus ergab eine leere Blase.

27. Sept. Patient etwas somnolent, phantasirt während der Nacht. Puls gehoben, Temperatur befriedigend, Zunge belegt, Stühle dünn, gallig, Katheterismus ergab ein negatives Resultat. Singultus. Die mit dem Katheter Morgens 8 Uhr entleerte Flüssigkeit besteht nur ans Eiter. Abends 7 Uhr werden mit dem Katheter $25 \mathrm{Ccm}$. Urin entleert, der sehr viel Eiweiss, sehr wenige Cylinder, viel Eiter und Blutkörperchen enthält. Chlornatr. $=0,5 \mathrm{p} . \mathrm{m} .=0,0012 \mathrm{Gr}$.

28. Sept. 'Patient sehr unruhig; PuIs und Temperatur befriedigend, Zunge reinigt sich, Stühle noch sehr dünn, gallig tingirt. Urin mit dem Katheter entleert, $140 \mathrm{Ccm}$, sauer, enthält noch immer eine ziemlich bedeutende aber gegen gestern geringere Quantität Eiweiss, die wenigen Cylinder sind aus ihm verschwunden. Gehalt an Harnstoff $14 \mathrm{pr}$. mll. $=4,06 \mathrm{Gr}$. Abends $6 \mathrm{Uhr}$ mit dem Katheter noch $150 \mathrm{Ccm}$. Urin entleert, Eiweiss in derselben Menge darin wie am Morgen, das Mikroskop zeigt wieder eine mässige Anzahl Cylinder und Epithelien, die mit Fetttroplen gefüllt sind. Eiterkörperchen.

29. Sept. Patient sehr unrubig, Puls frequent und voll, Temperatur erhöht, Gesicht geröthet, ebenso die Zunge, Leib gespannt aber nicht schmerzhaft. Stühle unter sich, gelb gefärbt. Klagen hat Pat. nicht zu fübren, er ist ziemlich somnolent. Urin mit dem Katheter entleert. $400 \mathrm{Ccm}$., sauer, enthält Eiweiss, geringe Flockenbildung, Epithelien und Eiterkörperchen; Cylinder sind in sechs Proben nicht zu entdecken. Gehalt an Harnstoff 17 pr. mll. $=6,8$ Gr.

30. Sept. Pat. sehr somnolent. Puls sehr voll, frequent, Temperatur erhöht. Conjunct. injicirt. Zunge noch belegt. Stïhle unter sich, gelbbraan. Urin mit dem Katheter entleert. $800 \mathrm{Ccm}$., sauer, enthält Eiweiss, mässige Flockenbildung, Epithelien, Eiterkörperchen, Cylinder in geringer Zahl. Gehalt an Harnstoff = 18 pr. mll. $=14,4 \mathrm{Gr}$.

1. October. Pat. noch somnolent, Puls voll, frequent. Temperatur erböht. Zunge sehr geröthet, trocken. Stïhle unter sich, gelb gefärbt, Urin mit dem Katheter entleert. $1160 \mathrm{Ccm}$, stark sauer, Eiweissgehalt, geringe Flockenbildung. Das Mikroskop zeigt eine Anzahl Eiterkörperchen (wie bei Blasenkatarrh), einzelne Epithelien. Gehalt an Harnstof $=17,5 \mathrm{p} . \mathrm{m} .=19 \mathrm{Gr}$.

2. October. Pat. noch sehr somnolent, Puls voll, mässig frequent, Temperatur erhöht, Zunge sehr trocken, geröthet, Stühle unter sich, Urin selbst entleert. $900 \mathrm{Ccm}$, sauer. Eiweissgehalt stark, Flockenbildung. Harnstoff $=16=14,4 \mathrm{Gr}$.

3. 0ctober. Der Zustand sehr verschlimmert; beschleunigte, sehr erschwerte 
Respiration, sehr kleiner Puls, ganz gesunkene Temperatur, vollständige Apathie. Tod 10 Uhr Morgens.

Vom Sectionsbefunde wollen wir nur das Wichtigste anfïhren: In der Niere umschliesst die Corticalis inselförmig die fettig entartete Medullarsubstanz, nur 2 bis 3 Pyramiden in jeder Niere reichen mit ihrer Papille bis in den Hilus. Im Darmkanal: starke Hyperämie, viele Peyer'sche Plaques und Follikel noch stark geschwollen.

3.

Schwere Asphyxie. Reichliche Transsudationen. 76 stündige Dauer der Anurie. Harnstoffauscheidung im Typhoid sehr spärlich. Tod.

August Lehmann (J. 356), Maschinenbauer, 32 Jahre alt, 24. August 1866

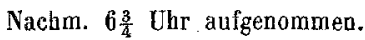

24. August. Patient gibt an, dass er heute Nacht plötzlich Diarrhoe bekommen habe, ohne Veranlassung; heut Morgen begann er zu erbrechen und zu collabiren; Diarrioe und Erbrechen haben fortwährend angedaverf; er will seit gestern Abend 7 Uhr keinen Urin mehr entleert haben. St. praes.: Pulslosigkeit, Temperatur gesunken, Haut mit Schweissen bedeckt, matschig; 0ppress. pector. Cyanose, heftige Wadenkrämpfe, Vox cholerica, hohlliegende Augen, grosser Collapsus, sehr beftiges Erbrechen. Ord.: Eis, Selter. Injection von Chinin. muriatic. Gr. viij.

25. August. Patient bat nicht geschlafen, Puls geboben, Temperatur fast normal, Stühle dünn, etwas gallig tingirt. Zunge belegt, Singultus. Erbrechen noch vorhanden. Conjunctiva injicirt.

26. August. Patient hat wenig geschlafen, Puls voll, Temperatur fast normal; Stühle dünn, braun gefärbt, Zunge trocken und belegt. Erbrechen cessirt. Ka theterisiren-ergibt ein negatives Resultat. Acid. muriatic. 8 Blatigel hinter den Ohren.

27. August. Patient hat etwas geschlafen; Puls voll, von Zeit zu Zeit aussetzend. Temperatur normal. Stühle dünn, braun gefärbt; Zunge reinigt sich. Urin nach 76 stïndiger Daver der Anurie selbst entleert. $525 \mathrm{Ccm}$. sauer, spec. Gew. 1,015, Eiweissgehalt bedeutend, Spuren von Gallenfarbstoff. Das Mikroskop zeigt: Schleimkörperchen, Epithelien, freie Zellen, Cyliader. Gehalt an Chlornatrium $=0,99 \mathrm{pr}$. mill. $=0,519 \mathrm{Gr}$. Gehalt an Harnstoff nach Ausällung des $\mathrm{E}$ iweisses $=15$ pr. mill. $=7,87 \mathrm{Gr}$. Der zweite Urin, um $11 \mathrm{Uhr}$ desselben Morgens gelassen, ist wieder stark sauer, $125 \mathrm{Ccm}$, Eiweissgehalt bedeutend, entbält dieselben milroskopiscben Gebilde, nur in geringerer Zahl. Gehalt an Chlomatrium $=1,55 \mathrm{pr}$. mill. $=0,19 \mathrm{Gr}$. Gebalt an Harnstoff $=19$ pr. mill. $=2,37 \mathrm{Gr}$.

28. August. Patient hat geschlafen. Puls freq. (96), Temperatur normal, Zunge etwas tracken. Stühle profus, sehr dünn, gelb gefärbt, Urin entleert. Conjunctiva injicirt. Urin in 24 Stunden $160 \mathrm{Ccm}$. sauer, spec. Gew. 1,016, viel Eiweiss und viel Gallenfarbstoff, das Milsroskop zeigt das gestrige Bild. Gebalt an Chlornatrium $1,7 \mathrm{pr}$. mill. $=0,27 \mathrm{Gr}$. Harnst off ge ba $\mathrm{lt}=19 \mathrm{pr}$. mill. $=3,04 \mathrm{Gr}$. 
29. August. Patient sehr unruhig. Puls frequent, in unregelmässigen Pausen aussetzend, Temperatur ziemlich gut, Stuhl noch sehr dünn, profus, gelb gefärbt. Abdomen auf Druck schmerzhaft, Zunge sehr trocken. Urin $240 \mathrm{Ccm}$., stark sauer, spec. Gew. 1,011, wenig Eiweiss, viel Gallenfarbstoff. Mikroskop zeigt wenige nicht von Epithel besetzte hyaline Cylinder, einzelne kernhaltige Plattenepithelien und runde granulirte Zellen. Gehalt an Chlornatrium $0,851 \mathrm{pr}$. mill. $=0,229 \mathrm{Gr}$. Harnst offgehalt $=19$ pr. mill. $=5,13 \mathrm{Gr}$.

30. August. Patient sehr unruhig. Puls aussetzend, Temperatur etwas erhöht. Stühle noch sehr dünn. Erschwerte Respiration. Zunge sebr trocken. Urin sauer, $100 \mathrm{Ccm}$., spec. Gew. 1,011, mässige Flockenbildung von Eiweiss. Das Mikroskop zeigt sehr viele durchweg schon mit Fetttröpfchen besetzte Cylinder, wenige entartete Epithelien, Eiterkörperchen und freie entartete Zelien. Gehalt an Chlornatr. $=0,99$ pr. mill. $=0,099 \mathrm{Gr}$. Gehalt an Harnst off $=18$ pr. mill. $=1,8 \mathrm{Gr}$, viel Gallenfarbstoff. Patient Nachmittag $2 \frac{1}{2}$ Ubr gestorben.

Aus dem Sectionsbefunde fübren wir nur an: Auf der Schleimbaut des Magens und Darmkanals grosse Blutextravasate. Peyer'scbe Plaques in ziemlicher Anzabl, grösstentheils bereits ausgefallen. - Nieren erheblich vergrössert, Kapsel leicht abziehbar; Corticalsubstanz gelblich, Pyramiden weissgelb, bis auf einzelne, die noch intact zu sein scheinen, fettig entartet.

So charakteristisch auch der abnorm niedrige Chlornatriumgehalt des Urins für den Choleraprozess ist, so wenig lässt er sich mit demselben in einen rationellen Connex bringen. Er ist immer abnorm niedrig, gleichviel ob die Transsudation nach dem Darmkanal lange oder kurze Zeit andauerte, ob das Erbrechen und die Dejectionen sehr profus waren oder nicht, ob in diesen Extreten vorher relativ und absolut viel oder wenig Chlornatrium ausgeschieden war, ob die Krankheit zum Tode führte oder mit rascher Genesung endete.

Die Chlornatriumausscheidung ist demnach nur für die Diagnose des vorangegangenen Gholeraprozesses, nicht prognostisch für den weiteren Verlauf der Krankheit zu benutzen.

Von der grössten Wichtigkeit für die Prognose ist die Dauer der Anurie. Fast alle Autoren stimmen darin überein, dass je früher die Urinsecretion wieder eintritt, desto sicherer ist die Genesung des Patienten. Aber wie lange darf die Anurie andauern, ohne dass die Hoffnung auf Genesung schwindet? Die Angaben der exactesten Beobachter gehen hierin weit auseinander. Die Einen theilen Genesungsfälle mit nach 4 - 5tägiger, die Anderen nach 6-7, ja 8tägiger Anurie. Sieht man die Fälle genauer an, so fehlt meist die Angabe, wann der Patient zuletzt Urin 
gelassen, und es scheinen jene Beobachter den Beginn der Anurie von dem Beginne der Krankheit zu datiren. Ueber den Beginn der Krankheit herrscht aber eine eben solche babylonische Verwirung; bei dem Einen beginnt die Krankheit schon mit dem ersten Durchfall, dern Anderen mit dem ersten Reiswasserstuhl, einem Dritten mit dem Erbrechen. Daher die Differenz der Angaben. Wir haben daher sowohl in der Epidemie von 1855 als auch in der letzten Epidemie bei jedem in's Lazareth eingelieferten Kranken die Zeit, wann er zuleizt Urin gelassen, soweit es sich zuverlässig ermitteln liess*), genau in's Journal notirt. Es sind in der Epidemie von 1855 im Lazareth von 509 Pat. 204 in der Asphyxie gestorben, haben also keinen Urin gelassen; 29 haben zwar die Asphyxie überwunden, sind jedoch an Urämie verstorben, ohne dass die Urinsecretion sich wieder eingestellt hätte. Von den übrigen 276 Pat. haben 39 am ersten Tage ihrer Erkrankung Urin gelassen, und sind sämmtlich genesen; 90 am zweiten Tage, von denen 84 genesen, also 93,3 pCt. und 6 gestorben, also 6,6 pCt.; 103 am dritten Tage, von denen 88 oder 85,4 pCt. genesen, und 15 oder 14,5 pCt. gestorben. 12 haben am vierten Tage, 2 am fünften und 1 am siebenten Tage den ersten Urin gelassen, und sind sämmtlich gestorben. Bei 29 Personen konnte die Urinsecretion mit Bestimmtheit nicht ermittelt werden.

In der letzten Epidemie sind 206 in der Asphyxie verstorben, ohne Urin secernirt zu haben. Von den ubrigen Asphyetischen sind leider nur 91 Fälle genau notirt. Am ersten Tage haben 28 Urin entleert und sind sämmtlich genesen; 35 am zweiten Tage, von denen 32 oder 91,4 pCt. genesen und 3 oder 8,5 pCt. gestorben; 17 am dritten Tage, von denen 13 oder 76,4 pCt. genesen und 4 oder 23,5 pCt. gestorben; 11 am vierten Tage sind sämmtlich gestorben. Eine Urinretention von länger als 4 Tage ist in der letzten Epidemie nicht beobachtet worden.

Von der Epidemie des Jahres 1853 können wir keine Zahlen liefern, da die Urinsecretion zwar genau beobachtet, aber nicht journalisirt worden ist, aber soweit unsere Notizen reichen, ist in jener Epidemie dasselbe Resultat in noch eclatanterer Weise her-

*) Die Angaben des weiblichen Geschlechts sind meist unzuverlässig und nur mit Vorsicht zu benutzen, da sie den Vrin zugleich mit dem Stuhl entleeren und daber meist glauben, mit jedem stuhl auch Urin entleert zu haben. 
vorgetreten. - Wir können demnach mit Bestimmtheit behaupten, niemals einen Genesungsfall in der Cholera gesehen $z u$ haben nach einer Urinretention von 3 mal 24 Stunden, und wir waren nach den Erfahrungen von 1853 in den beiden letzten Epidemien in allen Fällen im Stande, nach genau beobachteter 72stündiger Dauer der Amurie, den lethalen Ausgang mit Sicherheit zu prognosticiren. So haben wir, wie in vielen anderen Fällen, in dem oben mitgetheilten dritten Falle, August Lehmann schon am 27. August, dem vierten Tage der Krankheit, obgleich Pat. sich relativ wohl befand, zum grossen Erstaunen einiger anwesenden Collegen, das lethale Ende mit Sicherheit prognosticirt. Bei Kindern traten nach so langer Anurie sehr bald urämische Erscheinungen auf und starben sämmtlich unter Convulsionen. Bei Erwachsenen folgte nicht immer Urämie, zuweilen ein leidlich befriedigender Zustand, selten stellte sich die Urinsecretion in erheblichem Maasse wieder ein, mit nur sehr geringem Harnstoffgehalt, aber der lethale Ausgang blieb in keinem Falle aus. Wir werden beim Typhoid ausführlich auf den Verlauf zurückkommen.

II. Die Aphonie müssen wir zu den constanten Symptomen der Asphyxie zählen, da wir sie nie in asphyctischen Fällen vermisst haben, von der Klanglosigkeit der Stimme bis zum gänzlichen Schwinden derselben. Wobl vermag der Asphyctische durch Willensimpuls zuweilen hellere Töne auszustossen, jedoch nur ein Moment. Wenn es sich um Stillung seines unlöschbaren Durstes handelt, hört man von dem kurzen Schrei nach "Wasser" nur die erste Silbe. Die Aphonie ist schwach in den transsudativen Fällen, am stärksten, wo wenig Flüssigkeit transsudirt wird. Die Theorie der Austrocknung der Kehlkopfsschleimhaut in Folge des grossen Wasserverlustes stimmt daher nicht mit der Wirklichkeit.

III. Die Pulslosigkeit ist zu allen Zeiten das Hauptmerkmal der Asphyxie gewesen, obgleich das Herz lange nach dem Verschwinden des Radialpulses noch meist in kräftigster Action ist. Es ist charakteristisch für die Cholèra, dass der Puls nicht maassgebend für die Herzaction ist. Es können Cholerakranke 1, ja 2, zuweilen 3 Tage pulslos leben, aber nur wenige, nach unserer Beobachtung in maximo 12, Stunden ohne jegliche Herzaction. Wir haben in der letzien Epidemie eine Frau in's Lazareth kommen sehen, die vollständig cyanotisch und pulslos einen 
halbstündigen Weg (von der Bergstrasse) ohne Begleitung zu Fuss zurücklegte. Bei der Untersuchung war der Spitzenstoss des Herzens deutlich sichtbar, beide Herztöne stark zu hören, keine Spur von Puls zu fühlen, trotzdem ging sie jetzt noch zwei Treppen zur Station allein hinauf. Nicht das Fehlen des Radialpulses, sondern eines oder beider Herztöne bestimmte daher unsere Prognose.

Von grossem Interesse ist das Verhältniss der Urinsecretion zur pulslosigkeit, vollkommen entsprechend dem engen Zusammenhange zwischen den Spannungsverhältnissen des Gefässsystems und den Secretionsvorgängen der Niere *). Tritt die Pulslosigkeit rapid auf, so hört die Nierenfunction gleichzeitig vollständig auf, in Folge der aufgehobenen Spannung im Aortensystem. Sobald aber der Radialpuls allmählich schwindet, so tritt bei allmählich sich vermindernder Spannung im Aortensystem, eine ungleich höhere Spannung im Venensystem ein, und in dieser kurzen Uebergangszeit wird noch eine geringe Menge eiweisshaltigen Urins entleert, den wir oben als den zuletzt gelassenen eiweisshaltigen Urin geschildert haben. Von der kurzen oder langen Dauer dieser Stau ungshy perämie der Niere, also der kurzen oder langen Dauer der Asphyxie, hängt es ab, ob im Reactionsstadium in Folge des erhöhten Blutdruckes die Urinsecretion früh oder spät wiedereintritt. Jedenfalls wird so lange als die venöse Stauung in den Nieren vorhanden ist, ein eiweisshaltiger Urin entleert. Mit der Abnahme der hohen Spannung im Venensystem, die sich an den peripherischen Theilen in dem Schwinden der Cyanose markirt, vermindert sich auch der Eiweissgehalt des Urins, die Gallertschläuche, welche bisher die Harnkanälchen verstopft hatten, werden noch mit Leichtigkeit eliminirt, die Nieren müssen jetzt die erböhte Function übernehmen, alle die während der Asphyxie angehäuften Excretionsstoffe nunmehr auszuscheiden, und die Harnstoffausscheidung im Urin steigert sich, wie schon erwähnt, auf 70-80 Gramme p. d. Bei lang er Dauer

*) Diese von uns im Jahre 1855 bereits beobachteten Verhältnisse sind uns erst durch die im Jahre 1856 von Traube veröffentlichte berühmte Schrift "Ueber den Zusammenhang von Herz- und Nierenkrankheiten" klar geworden. Mit Recht verweist Griesinger in seiner klassischen Monographie nachdrücklich auf jene Arbeit Traube's. 
der Stauungsbyperämie treten fettige Degenerationen der Epithelien ein, es kommt zum theilweisen Schwund der Harnkanälchen, so dass die Nieren kaum die normale, viel weniger jene erhöhte Function übernehmen können. -

Hieraus entwickeln sich jene secundären Krankheitserscheinungen, die man, so mannigfach auch die Organe sind, welche vorwiegend dabei betheiligt sind, unter dem Collectivnamen Choleratyphoid zusammengefasst hat. Ob mit Recht oder Unrecht, ist ein müssiger Streit; auf das Wort kommt es nicht an, wenn man nur den richtigen Begriff damit verbindet. Vorweg muss man vom Choleratyphoid jene Zustände treanen, welche schon vor dem Choleraanfall im Organismus begründet waren, nunmehr nach demselben in verstärklem Maasse auftreten und zum Tode führen. Tuberculose, chronische Katarrhe machen sich im Choleraanfall nicht bemerklich, der quälende Husten, die profusen Sputa sind spurlos verschwunden, und kaum ist das asphyctische Stadium überwunden, treten jene Prozesse floride auf und führen rasch zum Tode. Auch alte schwächliche Subjecte können oft die Asphyxie überleben, und gehen dann an Erschöpfung zu Grunde. In diesen und ähnlichen Fällen spielt die vorangegangene Anurie so wenig, wie die wiedereingetretene reichliche Urinsecretion eine Rolle; der Verlauf hat durchaus nichts Charakteristisches.

Bei sonst gesunden kräftigen Individuen aber können nach der Asphyxie Krankheitserscheinungen ảuftreten, die wirklich als Folgezuständedes Choleraprozesses zu betrachten sind, und trotz der Verschiedenheit des vorwiegenden Localleidens doch einen gemeinsamen Charakter tragen. Es ist diess jene Depression des Nervensystems von leichter Somnolenz, die selbst bei. rascher Genesung nicht fehlt, bis zum tiefsten Sopor, aus dem der Kranke zwar meist noch zu erwecken ist, aber bald in denselben zurückfällt. Die Harnstoffuberladung des Blutes und der Gewebe in der Asphyxie macht sich beim Eintritt der Blutcirculation geltend. In den transsudativen Fällen ist ein Theil des Harnstoffs bereits während der Asphyxie in dem Erbrochenen als Harnstoff, in den Dejectionen als kohlensaures Ammoniak aus dem Körper entfernt worden, und daher gehen diese Fälle nach unseren Beobachtungen leichter in rasche Genesung über als jene, bei denen die profusen Transsudationen fehlten; aber diese doch nur verhältnissmässig ge- 
ringe Harnstoffausscheidung ist keineswegs ausreichend, es können immer noch nervöse Symptome auftreten, bis die Harnstoffausscheidung im Urin ihr Maximum erreicht hat. Hauptsächlich aber hängen die leichten oder schweren typhoiden Zufälle ab von der kurzen oder langen Dauer der Anurie und der quantitativen und qualitativen Beschaffenheit der folgenden Diurese. Gehen wir von den ächt urämischen Fällen aus, deren Beziehung zur sistirten Diurese von den wenigsten Beobachtern noch angezweifelt wird, so ist ihre Häufigkeit in den einzelnen Choleraepidemien verschieden. Im Jahre 1853 sind von 32 typhoiden Fällen 20, also 62,5 pCt., unter urämischen Erscheinungen gestorben, und 12 leichiere typhoide bei wiedereingetretener reichlicher Urinsecretion genesen. Im Jahre 1855 haben wir unter 107 Typhoidfällen 53, also 49,5 pCt, Urämische beobachtet, die meist unter Convulsionen starben. Unter diesen hat sich bei 29 die Urinsecretion gar nicht wieder eingestellt, bei 12 Urämischen erst am vierten Tage, bei 2 am füntten Tage, bei 1 Pat. am siebenten Tage und endlich in 6 Fällen zwar zu Ende des dritten Tages, aber die Urinsecretion blieb sehr spärlich mit zunehmendem Eiweissgehalt bis zum Tode. In den letzten Fällen sind Convulsionen nicht beobachtet worden. Bei 3 Urämischen konnte die Urinsecretion mit Bestimmtheit nicht festgestellt werden.

In der letzten Epidemie haben wir unter 120 Typhoiden nur 5 exquisit urämische Fälle beobachtet, bei denen die Urinsecretion erst nach 3tägiger Dauer der Anurie sich einstellte, und in spärlicher Quantität (von 300-1000 Ccm.) mit fortdauerndem starken Eiweisgehalt, und geringer Harnstoffausscheidung (von 4-20 Grm. p. d.) bis zum Tode blieb. Zwei Fälle derart haben wir in Tab. IV u. $\mathrm{V}$ skizzirt mitgetheilt.

Die Symptomenreihe war in allen Epidemien dieselbe. Benommenheit des Kopfes, grosse Schlafsucht, wenig Klagen über Beschwerden, nicht einmal über Kopfschmerz, selten Erbrechen grüner Massen, Amblyopie und Amaurose ist bei dem Zustande der Patienten nicht zu ermitteln, sie wissen weniger Auskunft. zu geben über ibre Gegenwart, als über die ihnen geläufige Vergangenheit. So hat ein urämischer Landwehrmanin auf die Frage, welche Schlachten er mitgemacht, stets bis an sein Ende die Orte Skalitz, Trautenau, Königsgrätz hergezählt, während er über seinen gegenwär- 
tigen Zustand keine Auskunft geben konnte. Oft grosse Unruhe, Neigung das Bett zn verlassen ohne Ziel und Zweck. Der Puls meist unregehnässig, nicht frequent. Die Respiration ist besonders charakteristisch. Die Inspirationen sehr tief, mühevoll, schon in der Ferne zu hören, die Halsmuskeln sind dabei sichtbar thätig, die Exspiration kurz, seufzenơ, oft blasend. Die Zabl der Inspirationen sinkt von 30 auf 20 herab. Die Schleimhaut der Lippen, Zunge und vordere Theil der Nase mit schwarzem Belag überzogen. Der Leib besonders in der Cöcalgegend beim Druck schmerzhaft, jedoch nicht constant. Dünne fäculente Stublentleerung meist unter sich. Der Tod wird meist nach $3-5$ Tagen durch Convulsionen des ganzen Muskelsystems herbeigeführt.

Der Schottin'sc̀he Belag ist in diesen Fällen nicht selten zu finden. Die Beschaffenheit des Urins, die mässige Quantität desselben, der geringe Harnstoff- und starke Eiweiss-Gehalt, die mässige Menge Cylinder in demselben, ist schon oben geschildert. In der Leiche findet man die Niere abnorm vergrössert, die Corticalsubstanz hat an Masse erbeblich zugenommen, so dass sie beim Schnitt in die Kapsel weit hervorquillt, die sonst geringe Distanz zwischen den einzelinen Pyramiden ist erheblich weiter und von gelblicher Corticalsubstanz ausgefült, die zuweilen einzelne Pyramiden ganz verdrängt bat, so dass ihre Papillen nicht mehr bis. an den Hilus reichen. Die Marksubstanz ist gelblichweiss und streifig wie Elfenbein, namentlich in der Mitte der Niere. Die seillichen Pyramiden bleiben lange dunkelroth und scheinen trotz der Degeneration der Epithelien die Secretionsfähigkeit lange conservirt zu haber.

Die zweite Reihe von Typhoidfällen ist nur eine mannigfache Modification des eben geschilderten Grundtypus. Dieser wird modificirt 1) schon durch die massenhafte Transsudation in der Asphyxie (s. o.); 2) durch die kürzere Dauer der Anurie; 3) durch reichliche Diurese und damit verbundene vermehrte Harnstoffausscheidung; 4) durch Hinzutritt anderer Krankheiten wie Diphtheritis im Darmkanal, Pneumonie, Pleuritis, Peritonitis, die fast immer letbal enden. Natürlich können die mannigfachsten Combinationen dieser vier Momente am Krankenbette eintreten. Wir haben alle möglichen Combinationen in den drei letzten Epidemien beobachtet, niemals aber den urämischen Grundtypus ver- 
misst. Jene Depression des Nervensystems, so wie die oben geschilderte charakteristische Respiration fanden wir in fast allen Fällen, natürlich in grösserem oder geringerem Grade, je aach der Combination der angeführten Momente.

In den zur Section gekommenen Fällen haben wir stets die Niere in ähnlicher Weise degenerirt gefunden, wie wir sie oben bei der Urämie gesehen haben. Wir müssen uns versagen, die hier einschlagenden genauen Krankengeschichten mitzutheilen und der Wichtigkeit des Gegenstandes angemessen für eine besondere Arbeit uns rorbehalten*).

Auch die Entzündung seröser Häute und des Lungenparenchyms dürfen nicht als gesonderte Complicationen betrachtet werden, sie stehen vielmehr in inniger Beziehung zu den Störungen der Urinsecretion, da sie ja auch bei Morb. Brightii so häufig auftreten**). Hier wie dort zeichnen sich diese entzündlichen Prozesse dadurch aus, dass sie ohne äussere Veranlassung ganz unerwartet sich entwickeln und sehr rapid reichliche Exsudation setzen. Wir haben Pneumonien beobachtet ohne Husten, obne Sputa, ohne Schmerzempfindung, nur die tägliche physicalische Untersuchung der Brust konnte dergleichen Affectionen entdecken, und dennoch sind wir zuweilen am Sectionstisch von einem grossen pericardialen eitrigen Exsudat überrascht worden, da intra vitam keine Erscheinung darauf hindeutete. Wir haben ein massenhattes pleuritisches Exsudat über eine ganze Brusthälfte durch Percussion entdeckt, das über Nacht sich entwickelt haben muss, da am Abend vorher keine Spur davon vorhanden war.

*) Rosenstein, Pathologie der Nierenkrankheiten S. 76, zweifelt in seiner sonst trefflichen Schilderung des Typhoids an den Zusammenhang desselben mit der Harnausscheidung, auf Grund eines von ihm citirten Falles. Aber wenn diese Krankengeschichte ohne Angabe der Quantität und Qualität des gelassenen Urins überbaupt etwas beweisen kann, so beweist sie das Gegentheil. Nur einmal, am 3ten Tage der Krankheit, war der Urin "reichlich", sonst stets "eiweisshaltig", meist "stark eiweisshaltig" bis zum Tode. Wenn man aus dem Eiweissgebalt auf den Harnstoffgehalt des Urins schliessen darf, so war in jenem Falle eine sehr geringe Harnstofausscheidung. Hierfür spricht auch der vielleicht urämische Krampfanfall vom 19. October, sowie der einzig erbebliche Sectionsbefund der Niere. Das Oedem der Pia mater findet man in asphyctischen wie typhoiden Fällen.

**; S. Frerichs, Bright'sche Nierenkrankheit. S. 131. 
Ebenso unvermerkt und rapid entwickeln sich die peritonealen Exsudate. Wir fanden nicht selten an der Leiche das Zwerchfell, den Peritonealïberzug der Leber mit flockigem eitrigen Exsudat bedeckt, ohne dass die Kranken nur eine Spur von Schmerz beim Druck auf jene Gegend angaben. Dieser Verlauf spricht entschieden für den innigen Zusammenhang der entzündlichen typhoiden Erscheinungen mit den Stockungen der Diurese, zumal sie sämmtlich lethal verlaufen.

Von den 120 Typhoiden der letzten Epidemie sind 50 in's Lazareth schon im typhoiden Stadium eingeliefert worden, ohne dass wir die Dauer der Anurie bestimmen konnten; von diesen sind 29 oder 58 pGt. gestorben und 21 oder 42 pCt. genesen. Von den übrigen 70 Typhoiden, welche die Asphyxie im Lazareth überstanden, sind 29 oder 41,4 pCt. gestorben und 41 oder 58,5 pCt. genesen. Es sind demnach von den 120 typhoiden Fällen 58 oder 48,33 pCt. gestorben und 62 oder 51,66 pCt. genesen.

Wir haben im Verlaufe des Typhoids sechs Fälle von Pneumonie beobachtet, und zwar 2 doppelseitig, 3 linkerseits und einmal rechterseits. Subjective Symptome fehlten meist in allen Fällen, nur einmal markirte sich der Eintritt der Pneumonie durch eine stärkere Frequenz des Pulses und der Respiration. Expectoration fehlte ganz bis zum lethalen Ende, das in keinem Falle ausblieb.

Zwei Fälle von Peritonitis konnten am Krankenbette nicht diagnosticirt werden, weil die Empfindlichkeit des Abdomen ganz unerheblich war, wenigstens nicht bedeutender als sie sonst nach einer schweren Asphyxie zu sein pflegt. -

Nimmt das Typhoid einen schleichenden Verlauf an oder zieht sich das Reactionsstadium in die Länge, so treten zuweilen Anschwellungen der Parotiden auf, welche aber den Krankheitsverlauf nicht sonderlich perturbiren. Im Jabre 1853 waren unter 32 Typhoiden drei Parotitiden, die in Eiterung übergingen, von denen nur ein Fall genas. Im Jahre 1855 unter 107 Typhoiden 3 Parotitiden, eine trat in einem Genesungsfalle im Reactionsstadium auf und 2 in schweren Typhoiden mit schleichendem, aber tödtlichem Verlaufe. In der letzten Epidemie traten die Parotitiden häufiger auf, unter 120 Typhoiden $9 \mathrm{mal}, 5 \mathrm{mal}$ doppelseitig, und zwar in 4 lethal verlaufenen Fällen und in einem Genesungsfalle; 
4 mal einseitig sämmtlich im protrahirten Reactionsstadium. Die Zeit des Auftretens schwankte zwischen dem 5ten bis zum 17ten Tage der Erkrankung. - In einem typhoiden Falle trat eine Entzündung der Sublingualdrüse ein, die in Eiterung überging und unterhalb der Zunge sich öfnete. Der Eiter reagirte, wie der der Parotiden, stark sauer.

Weit schlimmer für den Verlauf der Krankheit ist das Auftreten des gangränösen Decubitus, der absolut lethal verläuft. Einen solchen Fall haben wir im Jahre 1855 und einen in der letzten Epidemie bei einer Puerpera beobachtet. Hingegen haben wir eine colossale Verjauchung des subcutanen Bindegewebes des ganzen Rückens vom Nacken bis zur Sacralgegend bei einem 60jährigen Manne in Heilung übergehen sehen.

Delirium tremens entwickelte sich in der letzten Epidemie bei 6 Potatoren unmittelbar nach der Asphyxie (worunter auch ein Frauenzimmer), ohne dass der Krankheitsverlauf dadurch sich verschlimmert hätte, nur einer starb, mehr an der protrahirten Asphyxie als am Delirium. -

In allen drei Epidemien bewährten die im Reactionsstadium beim Uebergang zur Reconvalescenz auftretenden Exantbeme ihren prognostischen Werth. Im Jahre 1853 war bei 7 Pat. Urticaria aufgetreten im ausgesprochenen Stadium der Reconvalescenz, von deren nur ein Fall, ap später eingetretenen Complicationen, letbal verlief. - Im Jahre 1855 war $9 \mathrm{mal}$ Urticaria mit günstigem und 2mal Roseola mit tödtlichem Verlaufe vorgekommen; ein pustulöser Ausschlag führte zur Genesung und ein Pemphigus zum Tode. In der letzten Epidemie sind von 12 Urticariafällen 2 lethal verlaufen, bei denen, wabrscheinlich in Folge einer Erkältung, Entzündungen seröser Häute auftraten. Die, Urticaria trat in Genesungsfällen meist am 5ten bis 7 ten Tage nach Eintritt der Urinsecretion auf, zur Zeit der stärksten Harnstoffausscheidung, bei Individuen von heller Hautfarbe, meist Frauen. Bei dunkler Hautfarbe wird das Exanthem wahrscheinlich of übersehen, da man nicht selten in solchen Fällen eine leichte Desquamation der Baut wahrnimmt. Eine nähere Schilderung des Choleraexanthems ist bei der vortrefflichen Darstellung von Reinhardt und Leubuscher (l. c. S. 469) kaum möglich. -

Icterische Färbung der Conjunctiva, meist auch des Ge- 
sichts, verbunden mit einer leichten Anschwellung der Leber, haben wir $6 \mathrm{mal}$ im Reactionsstadium beobachtet. In allen Fällen war der Stuhl träge, aber nicht entfärbt, ebensowenig war im Urin eine erhebliche Menge Gallenfarbstoff nachzuweisen. -

Auf die weiblichen Genitalien scheint die Cholera stark zu influiren. Wir haben bei einem 25jäbrigen Mädchen nach der Cholera zum ersten Mal die Menses auftreten sehen, nachdem seit ihrer Pubertät ärztlicherseits alle Mittel zur Hervorrufung derselben vergebens angewandt wurden; die Menses traten in diesem Falle später regelmässig ein. Viel häufiger siebt man bei alten Frauen in den climacterischen Jahren die Menses nach der Cholera wieder eintreten, nachdem sie Jahre lang bereits cessirt haben. Das Blut gleicht dem Mestrualblute sowohl durch seine flüssige Beschaffenheit, wie seine dunkle Farbe und allmähliches Erblassen desselben, so dass zuletzt nur ein blutig tingirter Schleim abfliesst. Die Frage, ob diese Blutung als Menstruation oder als eine pathologische Hămorrhagie zu betrachten sei, ist schwer zu entscheiden, da diese Fälle gewöhnlich in Genesung übergehen und man daher keine Gelegenheit hat, die Beschaffenheit der Graaf'schen Bläschen zu eruiren. In den wenigen zur Section gekommenen Fällen derart, haben wir geplatzte und mit Blut gefüllte Graaf'sche Follikel nicht vermisst. Für einen der Menstruation mindestens ähnlichen Vorgang spricht auch der Umstand, dass bei jüngeren 12-14jährigen Individuen vor dem Erwachen der Geschlechtssphäre keine. Spur von Veränderung im Uterus und den Ovarien sich findet, nicht einmal jene so charakteristischen Blutextravasate in der Uterushöhle.

Einen weit schädlicheren Einfluss hat die Cholera auf den schwangeren Uterus. Es gebört zu den grossen Seltenheiten, dass eine Schwangere die Asphyxie durchmacht, ohne zu abortiren. Wir haben in drei Epidemien nur einen Fall derart gesehen (Journ. 506), Lina Abraham, 21 Jahre, Gravida im 2ten Monat, die eine heftige Asphyxie durchgemacht, aber von nur 24stiindiger Dauer und genas ohne Abortus, aber auch ohne Typhoid. Leichter e Cholerafälle kommen häufiger ohne Abortus davon, nicht blos bis zum 5ten Monat (Bouchut, Drasche, Baginsky), sondern auch daruber hinaus. Im Jabre 1855 ist uns eine Gravida im 9 ten Monat aus dem Arbeitshause eingeliefert worden, Marie Soltmann 
(Journ. 475) 39 Jahre alt, Puls und Temperatur sehr gesunken, Collapsus mässig, Erbrechen und Reiswasserstühle sehr profus, obne Typhoid genesen und nach 16 Tagen obne Abort entlassen. In der letzten Epidemie 2 Fälle: Minna Kraft (Journ. 318), 21 Jahre alt, Gravida im 7ten Monat, leichter Anfall ohne Typhoid nach 10 Tagen ohne Abort entlassen. (Journ. 495) Caroline Alexander, 28 Jahre, Gravida im 8ten Monat, leichter Anfall, erster Urin eiweisshaltig, Epithelien und Cylinder in geringer Menge; kein Typhoid, nach 8 Tagen ohne Abort entlassen. -

Das ungünstigste Mortalitätsverhältniss der Schwangeren war im Jahre 1855. Von 10 Schwangeren und einer post partum Eingelieferten sind nur zwei genesen, und zwar sind 4 in der Asphyxie verstorben und unmittelbar nach dem Tode durch Sectio caesaria entbunden, eine war im 5ten und $3 \mathrm{im} 9$ ten Monat; drei Schwangere (3 M., 5 M., 7 M.) abortirten im typhoiden Stadium und starben; 1 Gravida im 9ten Monat wurde entbunden in der protrabirten Asphyxie und starb; ein leichter Fall ist von einem 8 Monat alten todten Kinde entbunden und genas, und ein leichter Fall von 9 Monat ist ohne Abort genesen (s. o.). Eine Puerpera ist am Tage der Erkrankung von einem lebenden Kinde entbunden und am 2ten Tage der Krankheit in der Asphyxie verstorben. -

Günstiger gestaltete sich die Mortalităt in der letzten Epidemie. Von 16 Schwangeren und einer Puerpera starben 11. 3 Schwangere (6 M., 8 M., 9 M.) starben in der Asphyxie und wurden durch Sectio caesaria entbunden; 1 Gravida (8 M.) wurde in der protrahirten Asphyxie entbunden und starb; bei 9 Schwangeren (4 M., 5 M., 6 M., 7 M., 7 M., 8 M., 8 M., 8 M., 10 M.) ist die Entbindung im Typhoid erfolgt und nur 3 ( 4 M., 7 M., 7 M.) sind hiervon genesen. In drei Genesungsfällen (2 M., 7 M., 8. M.) ist kein Abort erfolgt (s. o.) und eine Puerpera starb an Pyaemie.

Es scheint demnach die Cholera kein Stadium der Gravidität zu verschonen, denn wenn auch die späteren Monate in den Angaben häufiger figuriren, so mag diess daher rübren, dass die ersten Monate der Gravidität meist erst durch die Ausstossung der Frucht zur Cognition des Arztes kommen, die späteren aber sofort entdeckt werden. - 
Die Ausstossung der Frucht geht meist sehr leicht vor sich, so dass die Placenta mit der Frucht in den unverletzten Eihäuten gleichzeitig zum Vorschein kommen. Nur einmal waren wir genöthigt, die Placenta nachträglich zu entfernen. - Fruchtwasser haben wir in allen Fällen in reichlicher Menge gefunden, namentlich da, wo wir durch Sectio caesaria die Frucht entfernen mussten. In den meisten Fällen wird die Frucht erst im typhoiden Stadium ausgestossen, selten schon in der protrahirten Aspbyxie, in keinem einzigen Falle haben wir sie auf der Höhe der Asphyxie beobachtet. -

Selbst in leichteren Fällen ist der Tod der Frucht sicher, herbeigeführt, wie wir glauben, durch den Choleraprozess. Wir haben in den drei letzten Epidemien 22 Kinderleichen sorgfältig obducirt und niemals jenen Befund vermisst, der, unserer Ansicht nach, für Cholera spricht. Man findet constant im Magen und oberen Theil des Dünndarms eine reiswasserähnliche Flüssigkeit, zuweilen eine concentrirte, aus abgestossenem Darmepithel bestehende Masse; Ecchymosen am Herzen fehlen nie; am hinteren Theil der Zunge sieht man schon Anschwellungen der Papillen, wie sie bei erwachsenen Choleraleichen in allerdings stärkerem Maasse anzutreffen sind. In der meist noch lappigen Niere markirt sich auffallend die gelbliche Cortical- von der blutreicheren Medullar-Substanz. Wenn auch Einzelheiten aus diesem Befunde bei anderen Kinderleichen vorkommen, so spricht dennoch der Befund in seiner Totalität, zumal er so constant vorkommt, für den fötalen Choleraprozess. -

Wir wollen hier die Lähmungen einzelner Muskelgruppen erwähnen, wie sie nicht gar häufig in dem Reconvalescenzstadium der Cholera vorkommen. Wir haben im Jahre 1855 fünf hierher gehörige Fälle beobachtet, und zwar 4mal Lähmung der Extensoren der Hand und $1 \mathrm{mal}$ des Sternocleidomastoideus der einen Seite. Jene 4 Fälle betrafen einen Mann und 3 Frauen im Alter von 23-37 Jahren, von denen 2 sebr leichte und 2 etwas schwerere Anfälle, jedocb keine Asphyxie durchgemacht haben. Es trat bei ihnen zwischen dem 7 ten bis 11ten Tage der Krankheit in voller Reconvalescenz eine Lähmung der Extensoren beider Hände, beim Manne blos der rechten Hand, ein, wodurch ein Uebergewicht der Flexoren, in Form einer Contractur, sich geltend machte. Die Stellung der Finger war analog der bei Bleilähmung 
beobachteten, und konnte hier wie dort mit Leichtigkeit in die normale Lage gebracht werden. Die permanent contrahirte Stellung der Finger war bei längerer Dauer schmerzhaft, verlor sich aber bald nach wenigen Tagen, ohne alle Medication. Der fünfte Fall betraf einen 3jährigen Knaben Hejnrich Schwengner (Journ. 231), sehr leichter Fall, am achten Tage der Krankheit Lähmung des rechten Sternocleidomastoideus, so dass der Kopf nach links in Form eines Caput obstipum hinübergezogen wurde. Im Jahre 1866 ist nur ein hierher gehöriger Fall vorgekommen bei einem 32jährigen Manne, der nach einem leichten Anfall 2 Tage nach seiner Entlassung mit einer Lähmung der Flexoren beider Hände wiederkam. Er ist aus Versehen nach der Charité dirigirt, so dass er von uns nicht weiter beobachtet werden konnte. Wir können diese Affection, nach Analogie der nach Diphtheritis eintretenden Paralysen, nur für eine Lähmung der Antagonisten, nicht für eine spastische Contractur der Flexoren halten. Ein Krampf würde nicht so permanent wirken, sondern auch zeitweise nachlassen. Das Auftreten dieser Affection nach leichten Choleraanfällen entspricht vollkommen dem Auftreten der Lähmungen nach leichter unbedeutender diphtheritischer Affection.

R e s u m é.

Werfen wir nunmehr in Berücksichtigung der eben geschilderten Thatsachen einen Gesammtblick auf den Choleraprozess, so müssen wir vorweg den Transsudationen die Wichtigkeit streitig machen, die ihnen seit langen Jahren beigelegt worden ist. Schon in den ersten Epidemien ist dem klinischen Scharfblicke Romberg's die bisher unbestrittene Thatsache nicht entgangen, dass grade die transsudativen oder ,enterischen“ Fälle, bei denen, wie wir gesehen haben 20000-30000 Ccm. Flüssigkeit im Erbrochenen und Dejectionen entleert wird, am günstigsten verlaufen, während anderseits die stets lethal verlaufenden sogenannten foudroyanten Fälle sehr unbedeutende Transsudationen liefern. Schon diese eine von keinem zuverlässigen Beobachter angezweifelte Thatsache sollte genügen, jene Theorie, welche die Transsudationen zum Angelpunkt des ganzen Choleraprozesses macht, zu erschüttern, zumal auch diese Theorie so wenig wie andere auch nur die wesentlichsten Choleraerscheinungen genügend zu erklären vermag. 
Aber man verweist uns bei jenen foudroyanten Fällen auf den bedeutenden Darminhalt post mortem! Nach unseren Messungen beträgt die Flüssigkeitsmenge im Darm in Fällen, die intra vitam wenig oder gar keine Ausleerungen hatten, 2-3000 Ccm. Um die maximale Darmcapacität zu ermitteln, haben wir einen vom Mesenterium getrennten, lang ausgezogenen Darmkanal eines Erwachsenen mit Wassèr prall gefüllt, und der Inhalt desselben betrug etwas über $6000 \mathrm{Ccm}$. Berechnet man den Widerstand, den der Darmkanal in seiner natürlichen Lage durch die Windungen und Bauchdecken findet, so dürften $2-3000 \mathrm{Ccm}$ : annähernd der maximale Werth des Darminhalts sein; eine Flüssigkeitsmenge, welche in ächt transsudativen zur Genesung führenden Fällen in wenigen Stunden entleert wird. Ist es nach dieser Theorie denkbar, dass in einer Reihe von Fällen ein Verlust von 2-3000 Ccm. Flüssigkeit eine vollkommene Eindickung des Blutes und Tod herbeiführt, während in einer anderen Reihe von Fällen bei einem zehnfachen Verlust von Flüssigkeit eine rasche Belebung der Circulation und Genesung eintritt? Woher ersetzen die Cholerakranken, deren Blut durch Transsudation eingedickt und zur Circulation unfähig gemacht ist, so raseh jene Flüssigkeitsmenge, um das Blut wieder circulationsfähig zu machen, da ja der Durst mit der Asphyxie schwindet und die Patienten im reactiven Stadium wenig oder gar nicht mehr trinken? Mit Recht verweist Griesinger*), in seiner klaren und gediegenen Weise, auf die Erscheinungen einer acuten Brucheinklemmung oder Darmperforation hin, welche oft ohne erbeblichen Stoffverlust in ganz kurzer Zeit ein vollständiges Sinken des Pulses, totalen Collapsus und graue Färbung der Haut sehr ähnlich der Cholera herbeiführen. Griesinger lässt daher jene Transsudationstheorie halb fallen, und betrachtet "die Herzschwäche als eine von der Darmerkrankung durch die Nerven vermittelte." Obgleich wir vom klinischen Standpunkte aus die Darmerkrankung nicht. für den Ausgangspunkt des ganzen Prozesses halten können, da der pathologische Befund im Darme, wie wir gesehen haben, durchaus nicht gleichen Schritt hält mit der Intensität der Erscheinungen am Krankenbette, so müssen wir uns dennoch vorläufig der Griesinger'schen Anschauung anschliessen, da sie dem gegenwärti-

*) Griesinger, Infectionskrankheiten S. 406. 
gen physiologischen Standpunkte vollkommen entspricht. Bekanntlich hat Bernstein*) in Verfolg jenes Goltz'schen**) Klopfversuches, wonach in Folge mechanischer Reizung der peripherischen Darmnerven durch Klopfen der Bauchdecken mittelst eines Spatels, jedesmal Stillstand des Herzens eintritt, durch sorgfältige Versuche an Thieren nachgewiesen, dass der Grenzstrang des Sympathicus Fasern enthält, deren Reizung reflectorisch a uf die Herznerven wirkt, und dass das Centrum der Hemmungsnerven nicht automatisch, sondern refleciorisch wirkt. Hiernach würde eine Reizung der Reflexfasern des Sympathicus reflectorisch zuerst auf den Tonus der Gefässnerven und dann bei stärkerem Reize auf das Centrum des Vagus einwirken. Hiermit wäre auch jene auffallende Erscheinung in der Cholera erklärt, dass vollkommene Pulslosigkeit längere Zeit vorhanden ist bei voller Herzaction (s. o.). Es wird zuerst der Tonus der Gefässnerven, und vor Allem der peripherischen alterirt, dann bei fortdauerndem Reize der Stillstand des Herzens dureh reflectorische Wirkung auf das Vaguscentrum allmählich herbeigefübrt.

Hier verlässt uns die genaue Kenntniss der physiologischen Function des Sympathicus, und wir wollen uns daher in weitere Hypothesen nicht ergehen, da sie ohne physiologischen Hintergrund keinen Werth haben. Wir sind seit der Epidemie des Jahres 1853 der Ueberzeugung, dass nur mit den Fortschritten der physiologischen Erkenntniss des Sympathicus die Erkenntniss des Choleraprozesses Hand in Hand gehen wird und muss. Kein pathologischer Prozess weist mit grösserem Nachdruck darauf hin, welche Bedeutung dem Sympathicus im Organismus gebührt, als die Asphyxie. Das häufig plötzliche Auftreten derselben bei ganz gesunden Individuen, der rasche Verlauf, die plötzlich eintretende Wendung des Krankheitsprozesses in Genesungsfällen, das Aufhören der secretorischen Function sämmtlicher Drüsen, die stete Contraction der Pupille während der Asphyxie (v. Graefe) u. s. w., kurz Alles deutet auf eine tiefe Alteration des ganzen vegetativen Nervensystems. Wie diese zu Stande kommt, wissen wir so wenig wie die Transsudationstheoretiker. -

*) J. Bernstein, Untersuchungen über den Mechanismus des regulatorischen Herznervensystems, Da Bois's Archiv 1864.

**) Goltz, Vagus und Herz. Dies. Archiv Bd. XXVI. 
Das sogenannte typhoide Stadium ist lediglich Produkt der Aufhäufung der excrementitiellen Bestandtheile im Organismus während der Asphyxie, welche um so stärker ist, je geringer die Transsudationen in der Asphyxie waren, je länger die Dauer der Anurie war, und endlich je sparsamer die Harnstoffausscheidung im Typhoid stattfindet. Diese drei Momente combinirt, produciren den höchsten Grad der typhoiden Erscheinungen, die Urämie. Fehlt das eine oder andere Moment, sind z. B. die Transsudationen sehr reichlich gewesen, oder hat die Anurie nur kurze Zeit angedauert, so modificiren sich die typhoiden Erscheinungen bis zu einer leichten Somnolenz. Bei allen typhoiden Zuständen aber ist der urämische Anstrich unverkennbar, selbst bei den accidentellen Entzündungen der pareuchymatösen Organe und der serösen Häute.

Zur Feststellung der Diagnose der Cholera in Beziehung zur Gholerine und anderen in den Symptomen ähnlichen Krankheiten (metallische Vergiftungen) gibt es keinen sicherern Anhaltspunkt als die Anurie. Hat man einmal die Blase mit dem Katheter sorgfältig entleert, so findet man während der Asphyxie keinen Tropfen Urin mehr vor*), während in der Cholerine und anderen Krankheiten sich neuer Urin in der Blase bald einstellt.

\section{Prog nos e.}

Aus dem Vorhergehenden geht zur Genüge hervor, dass in der Asphyxie die profusen Transsudationen keine ungünstige Prognose gewähren, dass vielmehr mit dem Aufhören des Erbrechens ohne Nachlass der übrigen Erscheinungen erst der Höhepunkt der Krankheit gegeben ist, jener paralytische Zustand des Darmkanals, in welchem die Dejectionen bei jeder Bewegung des Kranken unfreiwillig abgehen. Je länger also das Erbrechen an-

*) Wir müsser hier auf einen möglichen Irthum aufmertsam machen. Es kommen Fälle vor, wenn auch sehr selten, die nach kurzer Daver des reactiven Stadiums in die Aspbyxie zurückfallen, bei denen man dann eine geringe Menge Eiweiss und Cylinder enthaltenden Urins findet, der in jenem kurzen Reactionsstadium in die Blase gelangt ist. Hat man einen Kranken mebrere Stunden nicht gesehen, so kann man jenes kurze Reactionsstadium äbersehen, und glaubt in der Asphyxie einen Cylinder enthaltenden Urin gefunden $z u$ haben. 
dauert, selbst bis ins reactive Stadium hinein, desto günstiger ist der Verlauf. - Von schlimmer Vorbedeutung ist die Oppressio pectoris, die auf der Höhe der Krankbeit selten fehlt, und bis zu Ende immer stärker wird. -

Wenn einerseits das $\mathrm{S}$ chwinden des Pulses am leichtesten die Asphyxie annoncirt, so bestimmt andererseits das Schwinden der Herzthätigkeit am sichersten die Prognose. Pulslose Kranke können immer noch genesen (in der letzten Epidemie $23 \mathrm{pCt}$.), während erst mit dem allmäblichen Schwinden der Herztöne die Prognose sich verschlechtert. - Dass blutige Stühle den tödtlichen Ausgang sichern, ist allgemein anerkannt. -

Viel sicherer lässt sich die Prognose im typhoiden Stadium stellen. Hat die Anurie länger als $3 \mathrm{mal} 24 \mathrm{Stunden}$ angedauert, so ist nach unseren Beobachtungen, der lethale Ausgang unaubleiblich. Ist die. Urinsecretion friher eingetreten, so. gewährt der Gehalt des Urins an Eiweiss, Cylinder und Harnstoff einen genügenden Anbaltspunkt. Je früher das E iweiss aus dem Urin schwindet, je zahlreicher die Cylinder in den ersten Urinen erscheinen, je reichlicher die Harnstoffausscheidung ist, desto günstiger ist die Prognose, und umgekehrt. Es sind diess so sichere Miomente, dass bei genauer Berücksichtigung derselben man die Prognose keineswegs verfehlen kann.

Complicationen jeder Art, Entzündungen parenchymatöser Organe und seröser Häute, ausgedehnter Decubitus, Gangrän etc. verschlimmern den Stand der Krankheit ebenso wie das Puerperium. Mässige Diarrhöen im Typhoid sind auch nach unseren Erfahrungen nicht unerwünscht. Dass die ächt urämischen Fälle stets lethal verlaufen, haben wir bereits erwähnt, namentlich die mit dem Schottin'schen Belag Behafteten.

\section{Therapie.}

Aus den früheren Epidemien haben wir die Ceberzeugung gewonnen, dass die trostlose Erfolglosigkeit aller unserer. Medication hauptsächlich auf Riechnung der überaus mangelhaften ResorptionsFähigkeit des Magens und Darmes während der Asphyxie zu bringen ist. Wir haben im Jahre 1855 die höchsten Dosen von Extr. nuc. vomic. spirit., von Strychn. sulph. innerlich gegeben, ohne eine 
schädliche Wirkung davon gesehen $z u$ haben. Ein Asphyctischer hat aus Versehen sogar $25 \mathrm{Gr}$. des Extr. nuc. vomic. spirit. innerhalb 48 Stunden verbraucht und ist ohne Anfechtung genesen. Wir schlossen hieraus, dass die Medicamente unresorbirt mit den Transsudationen aus dem Körper entfernt werden, und wandten uns daher in der letzten Epidemie von vorn berein der hypodermatischen Methode $\mathrm{zu}^{*}$ ), um die Arzneimittel auf diesem neuen Wege sicherer dem Organismus einzuverleiben. Die Beschaffenheit der Haut in der Asphyxie rechtfertigte den Zweifel an die Resorptionsfähigkeit derselben, der noch erhöht wurde durch das häufige Gangränesciren der Injectionsstellen.

Die durch Morphiuminjectionen erzielten temporären Erfolge bei Wadenkrämpfen waren nicht geeignet, die Resorptionsfrage der Haut in der Cholera endgültig zu entscheiden. Injicirt man nämlich Morphium in die Wadengegend, so wird allerdings nach kurzer Zeit die Schmerzempfindung auf ein Minimum reducirt, aber die Wirkung scheint Anfangs doch nur eine locale zu sein, denn die Wadenkrämpfe cessiren nicht, sobald die Injection am Oberschenkel gemacht wird. Auch wird hierbei die geballte Contraction der Wadenmuskeln, sowie die eigenthümliche Stellung des Fusses nach Art des Pes equinus in keiner Weise verändert. Es wird demnach, wie es scheint, nicht der Krampf beseitigt, was bei einer allgemeinen Wirkung der Fall sein müsste, sondern die Schmerzempfindung local und temporär vermindert. Die später eingetretene allgemeine Wirkung des Morphium lässt sich von den Krankheitserscheinungen schwer trennen und liefert daher keinen genügenden Beweis für die Resorption des Mittels.

Um nun die hypodermatische Resorption der Stoffe im asphyctischen Stadium der Cholera unzweifelhaft nachzuweisen, haben wir in 12 asphyctischen Fällen eine Quantität Ferro-Cyankalium subcutan injicirt, ein Salz, das im normalen Zustande bekanntlich schon nach 15 Minuten im Urin durch Eisenchlorid nachzuweisen ist. Da die Dejectionen, wie wir gesehen haben, ihr flüssiges Material, sowie ihren Gehalt an anorganischen Salzen dem Blute entnehmen, mussten wir voraussetzen, dass das injicirte

*) S. unsere vorläufige Mittheilung in der Berliner Klinischen Wochenschrift No. 35. 1866 . 
Blutlaugensalz im Falle der Resorption sich in den Dejectionen mit Leichtigkeit wird nachweisen lassen. Aber in keinem der 12 Fälle war das Blutlaugensalz bei der genauesten Untersuchung zu finden, weder in den Reiswasserstühlen noch in dem Erbrochenen. In den Fällen, die tödtlich verliefen, untersuchten wir genau die Injectionsstelle, macerirten das umliegende Zellgewebe und fanden meist nur Spuren des Salzes. Es war also zum grossen Theil resorbirt. In Fällen, welche die Asphyxie überwunden haben, kam das Blutlaugensalz im ersten, spätestens im zweiten Urin deutlich zum Vorschein. Aber dieser letztere Umstand bewies noch immer nicht die Resorption des Salzes in der Asplyyie, da dasselbe erst im Beginn des reactiven Stadiums bei der Neubelebung der Circulation resorbirt sein konnte.

Wir gingen daher zur Injection des Jodkalium über, welches sich nicht bloss im Urin, sondern schon nach 11 Minuten bei normalem Zustande auch im Speichel mit Leichtigkeit nachweisen lässt. In den Dejectionen konnte auch das Jodkalium nicht entdeckt werden, wohl aber fanden wir nach $3-\mathbf{5}$ Stunden in dem in der Asphyxie uns kärglich zugemessenen Speicbel deutliche Reaction des resorbirten Jodkalium.

Die räthselhafte Erscheinung, dass die injicirten Salze bei nunmehr unzweifelhafter Resorption derselben, dennoch in den Dejectionen nicht aufzuweisen waren, konnten wir nur auf den Umstand zurückführen, dass in den Choleradejectionen überbaupt nur Natronsalze aufufinden sind (Chlornatrium, phosphorsaures Natrium), die Kalisalze hingegen im Blute zurïckgebalten werden. Es wäre demnach möglich, dass in obiger Versuchsreihe das Kaliumeisencyanür und Jodkalium nur eben als $\mathrm{Kalisalze}$ im Blute zurückgehalten waren una desshalb in den Dejectionen nicht aufgefunden werden konnten.

Um die Richtigkeit dieser Erklärung nachzuweisen, injicirten wir in mehreren schwer asphyctischen Fällen Jodnatrium und nach $1 \frac{1}{2}$ Stunden war das Jod im Erbrochenen und Dejectionen nachzuweisen, im Speichel schon nach einer Stunde. -

Noch eclatanter erwies sich die Resorptionsfähigkeit der Haut in der Asphyxie bei Einspritzungen von Atropin. Fünf Tropfen einer Atropinlösung (Gr.j) Dr.j an den Oberarm injicirt, zeigten in einigen Fällen schwerer Asphyxie schon nach 15 Minuten, in 
anderen erst nach einigen Stunden Erweiterung der Pupille, während die doppelte Dosis per os eingebracht, selten die Wirkung herrorbrachte.

Aus diesen Versuchen geht unzweifelhaft hervor, dass selbst in der schwersten Asphyxie hypodermatisch injicirte Stoffe resorbirt werden, jedoch in einer viel langsameren trägeren Weise als im normalen Zustande. -

Hieraus folgt aber auch, dass man in sehr rapid verlaufenden Fällen und bei weit vorgeschrittener Asphyxie sich auch von der subcutanen Injection Nicbts versprechen darf, da immerhin 2-5 Stunden zur Resorption nöthig sind. Auch gibt es Fälle von sehr matschiger collabirter Haut, bei denen wahrscheinlich gar keine oder nur sehr geringe Resorption stattfindet, namentlich wo die Anästhesie der Haut so bedeutend ist, dass der Stich der Injectionsspritze nicht mehr gefühlt wird. Dieser letztere Umstand gab uns Veranlassung, in all diesen Fällen das letbale Ende mit Sicherheit zu prognosticiren. -

Die Mittel, die wir subcutan injicirt haben, waren der Reihe nach folgende:

1) Morphium hydrochlorat. (Gr. $\frac{1}{16}-\frac{1}{8}-\frac{1}{4}$ ) hauptsächlich gegen die beiden lästigsten Symptome, die Wadenkrämpfe und die Oppressio pectoris. Es ist nicht zu leugnen, dass meist schon sehr rasch (nach 10 Minuten) die locale Wirkung eintritt, die Verminderung der Schmerzempfindung. Nachdem aber später die volle Resorption stattgefunden, tritt auch die allgemeine Wirkung ein, eine für die Umgebung der Patienten höchst wohlthuende Euphorie, die aber, unserer Ansicht nach, den Patienten selbst rascher seinem Ende entgegenführt. Jene Lähmungserscheinungen des Nervensystems, die schon in dem Krankheitsprozess selbst liegen, werden durch diess Mittel noch gesteigert. - Wir haben daher die Morphiuminjectionen sehr bald aufgegeben, zumal jene drei lästigen Krankheitserscheinungen, gegen welche sie hauptsächlich angewandt werden, Wadenkrämpfe, Singultus und Oppressio pectoris, auf eine weit einfachere unschädlichere Weise zu beseitigen sind, nehmlicb durch locale Anwendung des Chloroform. Man lege, wie wir gleich hier mittheilen wollen, ein mit Chloroform getränktes Löschblatt auf die Waden, Magengegend oder Brust und bedecke diess rasch mit einer 
in warmes Wasser getauchten dicken Compresse, um die Verdampfung des Chloroform zu verhindern. Sehr bald röthet sich die Haut intensiv, der Singultus schwindet, die Schmerzempfindung an den Waden vermindert sich, die Oppressio pectoris wird weniger lästig, da sie, in dem Krankheitsprozess begründet, nicht gänzlich zu beseitigen ist. Wir ziehen diese Methode den Morphiuminjectionen vor, weil die locale Anwendung dieses flüchtigen Anästheticums keinerlei allgemeine Wirkung hervorbringen kann. -

Um den Choleraprozess selbst therapeutisch zu bekämpfen, haben wir auf subcutanem Wege hauptsächlich vom $\mathrm{C} h$ inin und Strychnin in ausgedehntern Maasse Gebrauch gemacht. Da wir uns in der Therapie der Cholera leider noch ganz auf dem schwankenden Boden des Experimentes befinden, so dürfen wir uns füglich jede Hypothese über den rationellen Zusammenhang jener Mittel zum Krankheitsprozess um so mehr ersparen, als naheliegende Motive für dieselben im Vorhergehenden hinreichend vorbanden sind. Wir haben also:

2) Ghinin. muriat. in der Dosis von Gr. ij-viij in 181 Fällen injicirt. Wir haben das Chinin. muriat. dem sulph. vorgezogen, weil man eine concentrirtere Lösung mit geringerem Zusatz von Säure vom salpetersauren Chinin anfertigen kann. Wir haben im Lazareth eine Solution von (Dr. j) Unz. $\beta$ zur Injection benutzt und die einzelne Dosis auf mehrere Injectionsstellen vertheilt, um nicht durch ausgedehnte Zerreissungen der Maschen des Zellgewebes die Nekrotisirung desselben zu befördern.

Nehmen wir auf den Grad der Krankheit Rücksicht, so stellt sich das Verhältniss folgendermaassen:

Bei 129 schwer Asphyctischen wurde Chinin injicirt, davon starben 84 oder 65,1 pCt. und genasen 45 oder 34,8 pCt. Von Ersteren haben noch 20 die Asphyxie überwunden und sind erst im Typhoid gestorben.

Bei 40 leicht Asphyctischen, bei denen der Puls verschwindend war, starben nach der Chinininjection nur 2 Personen oder 5 pCt. und genasen 38 oder 95 pCt.

Die übrigen 12 mit Chinininjection Behandelten befanden sich in der sogenannten protrahirten Asphyxie. Von diesen starben 8 oder 66,6 pCt. und genasen 4 oder 33,3 pCt.

3) Strychnin. sulph. (Gr. $\frac{1}{T^{2}}-\frac{1}{3}$ ) wurde injicirt bei 
61. Fällen der schwersten Asphyxie. Wir haben die verzweifeltsten Fälle zur Strychnininjection gewählt, weil wir bei einem 10 jährigen Kinde, das uns in ultimis eingeliefert wurde, nach Injection von Gr. $\frac{1}{3}$ Strychnin die heftigsten Reflexkrämpfe haben eintreten sehen. Von diesen 61 Fällen starben 51 oder $83,6 \mathrm{pCt}$. und genasen 10 oder 16,3 pCt. -

obgleich in den Lazarethen der früheren Epidemien bei keiner Kurmethode ein so günstiges Heilresultat erzielt worden ist, so wäre es dennoch voreilig, aus obigen Thatsachen allein auf die günstige Wirkung der Chinininjection zu schliessen, da es ja hauptsächlich auf den Charakter jeder einzelnen Epidemie ankommt. Wir sind in der letzten Berliner Epidemie in der glücklichen Lage, die Resultate der verschiedensten Heilmethoden in vier gleich situirten Lazarethen mit einnnder vergleichen zu können. Erst diese Vergleichung, die uns jetzt noch nicht zugänglich ist, wird uber den Werth der einen oder anderen Heilmethode und Heilmittel endgültig entscheiden können. Bis dahin müssen wir unser Urtheil über obige therapeutische Versuche aufschieben.

Einen therapeutischen Fortschritt aber im Vergleiche zu den früheren Epidemien glauben wir schon jetzt registriren zu können. Während wir in früheren Epidemien therapeutisch fast ausschliesslich auf den Magen und Darmkanal angewiesen waren, deren Resorptionsfähigkeit in der Asphyxie mehr als problematiseh erscheint, haben wir jetzt in der hypodermatischen Methode einen weit sichereren Weg zur Einführung der Mittel in den asphyctischen Organismus*).

Zu Ende der Epidemie kamen wir auf den Gedanken, Injectionen in die Harnblase zur Einführung von Medicamenten in's Blut der Asphyctischen zu benutzen, da in der Cholera die Blase das einzige mit Blutgefässen reichlich versehene Organ ist, welches vom Krankbeitsprozess wenig oder gar nicht turbirt wird. Die Blase ist in der Asphyxie stets leer und die reichliche Abstossung ihres Epithels in der Cholera spricht noch nicht gegen die Resorptionsfähigkeit derselben. Um diese zu prüfen, stand uns nur noch Ein asphyctischer Fall zu Gebote, den wir zu folgendem

*) Ich glaube, dass Euleaburg jun. zuerst auf die Anwendung der. hypodermatischen Methode in der Cholera bingewiesen hat. 
Versuche benutzten. Nachdem wir mittelst Katheter die Blase vollkommen leer gefunden hatten, injicirten wir in dieselbe eine vorher genau titrirte Lösung von Chlornatrium, die $45 \mathrm{p}$. mll. Chlornatr. enthielt, und nach einer Stunde entleerten wir diese Flüssigkeit mit dem Katheter. Diese war noch vollständig frei von allen Farbstoffen und mikroskopisch waren nur einige Epitbelien und Eiterkörperchen zu entdecken; ibr Gehalt an Chlornatrium ąber war nicht mehr 45, sondern nur noch 36 p. mll. Darauf injicirten wir abermals eine Lösung von $45 \mathrm{p}$, mll. Chlornatriumgehalt und entfernten die Flüssigkeit erst nach $z$ wei Stunden, sie enthielt nur noch 32 p. mll. Chlornatr., ohne alle urinösen Bestandtheile. Als wir aber diese Procedur zum dritten Male wiederholten und die Flüssigkeit nach 3 Stunden entfernten, enthielt sie bereits Harnstoff, Eiweiss und Cylinder. Es war also eine Quantität ersten Urins der Flüssigkeit beigemischt, so dass nunmehr die Verminderung des Chlornatr.-Gehalts nicht mehr zu bestimmen war. Schon aus diesem einen Versuche geht hervor, dass Stoffe durch die Blasenwandung in der Asphyxie resorbirt werden und es bleibt einer späteren Epidemie vorbehalten, zu prüfen, ob die Resorption der Harnblase in der Asphyxie nicht lebhafter und reger ist als die des Unterhautzellgewebes, da letztere doch noch Vieles zu wünschen übrig lässt. Eine wichtige Reihe von Versuchen haben wir mit der Anwendung des constanten Stromes gemacht. Hr. Dr. Fliess, dessen wissenschaftliche Befähigung und Exactität in diesem Fache allgemein anerkannt ist, hatte auf meinen Wunsch die Güte, in unserem Lazareth mit grossem Aufwand von Zeit und Mühe die Versuche selbst zu leiten. Vier der schwersten asphyctischen Fälle haben wir zu diesen Versuchen ausgewählt und obgleich sie alle lethal verliefen, können wir uns dennoch nicht versagen, von diesen für die Electro-Therapie in der Cholera so wichtigen Versuchen Einiges kurz mitzutheilen:

Ernst Jacob, Pustillon, 22 Jahre alt, 31. Juli Nachmittags $3 \frac{1}{2}$ Uhr aufgenommen. Patient, ein kräftiges muskulöses Individuum, erkrankte heute früh ohne Veranlassung an Durchfall. Bis Mittag hat er noch seinen Dienst versehen, bis Erbrechen eintrat. St. praes.: Pulslosigkeit, Temperatur gesunken, Oppress. pectoris, starke Cyanose, heftige Wadenkrämpfe, Vox cholerica, bedeutender Collapsus. - Um $6 \mathrm{Uhr}$ wurde der a ufsteigende constante Strom angewandt, 
indem die negative Electrode auf das Ganglion suprem. sympath. (zwischen Angul. maxill. infer. und Process. mastoideus) und die positive auf den Plexus cardiacus (zwischen 2te und 3te Kippe links vom Sternum) gesetzt wurde; Herztöne waren vor der Einwirkung noch ziemlich deutlich hörbar. Während die negative Electrode auf der rechten Seite $\frac{1}{4}$ Stunde einwirkte (40 Elemente), wurde auf derselben Seite der.Puls der Radialis fühlbar, während die linke Radialis nicht z a fühlen war. Das umgekehrte Phänomen zeigte sich, nachdem die negative Electrode auf der linken Seite 15 Minuten eingewirkt. Nach Verlauf dieser Zeit. war der 2te Herzton fast vollständig geschwunden, der 1 te schwächer geworden, obgleich man, während der Einwirkung des electrischen Stromes eine Verstärkung des 1 ten Herztones constatirt hatte; die Stellen, wo die Electroden eingewirkt, waren intensiv geröthet, und selbst Escherabildung bemerkbar. Während des Electrisirens trat mehrere Mal Erbrechen auf, das schon vorber cessirt hatte. Um 7 Uhr wurde Pat, in einem Betttuch gehüllt, in ein warmes mit 2 Pfund Salz versetztes Bad $\left(30^{\circ}\right.$ R.) gesetzt. Der positive. Pol wurde mit der Zinkwanne verbunden, während der negative, abgezweigt durch 2 Electroden, auf das Gang. supr. symphat. dextrum und sinistrum zu gleicher Zeit einwirkte. Pat. klagte Anfangs über heftiges Brennen von Seiten des Wassers. Nach 15 Minuten war der Puls in beiden Radiales deutlich fühlbar, er wurde allmählig voller, aber sehr frequent, kaum zählbar. Zeitweise nahm die Pulswelle an Höhe ab, blieb jedoch in ihrer Frequenz constant. - Das Bad dauerte eine Stunde, während welcher Zeit der Strom unablässig auf den Pat. einwirkte. Fin sichtliches Wohlbehagen war am Pat. bemerkbar, die Oppress. pectoris vermindert. Nach Ablauf einer Stunde konnte man wiederum deutlich ein Schwächerwerden des Pulses constatiren. Der Pat. wurde daher ans dem Bade genommen, und in wollese Decken zum Nachschwitzen gehültt. Die Auscultation ergab kräftige Herztöne, die beide vor dem electrischen Bade kaum hörbar waren; Cyanose nicht verringert. $\frac{1}{2}$ Stunde nacbher musste Pat. aus den Decken entfernt werden, da sich wiederum heftige Oppressio pectoris und grosse Unruhe eingestellt hatte. Gesicht mit kalten Schweissen bedeckt, Pols beiderseits nicht fühlbar, Herztōne kaum hörbar. Der Tod erfolgte um $9 \frac{1}{4}$ Uhr.

In einem 2ten ähnlichen Falle war dieselbe Procedur am folgenden Tage vorgenommen. Vor dem Bade war kein Puls zu fühlen, von den Herztönen nur einer schwach hörbar. Schon nach zehn Minuten war der Puls an beiden Radiales fühlbar, nach 20 Minuten noch deutlicher, die Herztöne deutlich börbar. Hier blieb die Circulation im Gange, der Patient starb erst am 6ten. Tage im Typhoid, da die Urinsecretion sich erst am 4ten Tage einstellte. -

Bei zwei anderen ähnlichen Fällen wurde der absteigende Strom mit gleichem noch schnellerem Erfolge angewandt, denn schon nach 3 Minuten hob sich der Puls und die Herzthätigkeit. 
Auch diese Patienten starben, der Eine noch in der Asphyxie, der Andere in der protrabirten Asphyxie.

Aus diesen wenigen Versuchen geht hervor, dass man in der höchsten Asphyxie mittelst des constanten Stromes noch im Stande ist, die geschwundene Herzthätigkeit, wenn a uch nur temporär, anzuregen. Es scheint auch eine allzulange Einwirkung des electrischen Stromes eher schädlich als nützlich zu sein. Leider sind diese Versuche so zeitraubend und mühevoll, dass sie in einer lebhaften Epidemie, wo die Thätigkeit der Aerzte im Lazareth nach allen Richtungen hin, bedeutend in Auspruch genommen wird, kaum durchzuführen sind. -

Nach den Anschauungen, die wir von den Darmtranssudationen im Vorhergehenden dargelegt haben, versteht es sich von selbst, dass wir weder gegen die profusen Dejectionen noch gegen das reichliche Erbrechen irgendwie therapeutisch agirt haben. Wir haben vielmehr in Fälen, wo das Erbrechen bereits cessirt bat, es wieder hervorzurufen versucht durch grosse Dosen Ipecacuanha wie durch subcutane Injectionen von Emetin, aber stets vergebens.

Ebenso haben wir uns fern gehalten von allen sogenannten Stypticis, z. B. dem Argent. nitric., das wir schon im Jahre 1855, selbst bei Kindern nutzlos gefunden haben. Wir baben damals 27 Kinder mit Arg. nitric. behandelt, davon genasen 8 oder 29,6 pCt. und starben 19 oder 70,3 pCt. Die 8 Genesenen waren leichtere Fälle. Bei den bedeutenden Ausscheidungen von Chlornatrium in den Darm geht sicherlich das Mittel als unlösliches Chlorsilber ohne alle Wirkung durch den Darmkanal. -

Auch dem 0 pium können wir nicht das Wort reden, da es den paralytischen Zustand des Darmkanals noch erhöht. Fast alle uns eingelieferten schwer Asphyctischen waren bereits mit Opium saturirt. Aber leider können wir in den Prodromalstadien das Opium nicht entbehren, so lange man den Unterschied zwischen Choleradiarrhoe und einfachem Intestinalkatarrh nicht kennt. Sobald aber wirkliche Choleraerscheinungen auftreten, sollte man sich hüten, das Opium anzuwenden. -

Wir haben in der letzten Epidemie, da man das Publicum mit der subcutanen Injection allein nicht zufrieden stellt, im Lazareth meist innerlich Calomel zu Gr.j mit oder ohne $\mathrm{Rheum}$ 
neben reichlichem Eiswasser verabreicht und glauben diesem Mittel das baldige Eintreten galliger Stühle vielleicht zuschreiben zu dürfen. -

Bei blutigen Stühlen haben wir Eisumschläge auf's $A b-$ domen und Eiswasserklystiere machen lassen, aber leider ohne Erfolg. -

Kalte Einwickelungen haben wir nicht selten als Unterstützungsmittel nicht ohne Erfolg benutzt.

Im reactiven Stadium sahen wir uns oft genöthigt, eine locale Blutentziehung am Kopfe zu machen, wo wir aus den Krankheits-Erscheinungen und der stark injicirten Conjunctiva (nicht zu verwechseln mit den in und nach der Asphyxie gewöhnlichen Conjunctivalstasen) Stasen im Gehirn vermutheten. Wir waren hierzu um so bereitwilliger, als wir häufig in diesem Stadium zur grossen Erleichterung der Patienten und mit bedeutendem Nachlass der Krankheitserscheinungen Epistaxis eintreten sahen.

Reichliches galliges Erbrechen hat uns auch in diesem Stadium zu keinem therapeutischen Eingriff bestimmen können, ebensowenig dünne gelbliche Stühle, so lange sie nicht in Rücksicht auf den Kräftezustand der Patienten allzu profus waren. In letztèrem Falle genügte meist ein Decoct. Colombo mit Acid. muriat., um sie zu mässigen. -

Wo die Urinsecretion reichlich von Statten ging, da konnte man von aller Therapie absehen und den Patienten sich selbst überlassen, wo aber die Diurese zu lange (länger als 72 Stunden) cessirt hatte oder die Harnstoffausscheidung zu spärlich war, da war auch unsere Therapie zu Ende. Diesen Zuständen gegenüber stehen wir hülf- und rathloser, als den asphyctischen. Keins der. angepriesenen Mittel ist im Stande, die Niere wieder zu restituiren, am allerwenigsten Reizmittel wie 0l. Terebinth. oder Juniperi. Nur leichtere typhoide Zustände können Gegenstand der Therapie werden und hier genügten meist schon Mineralsäuren oder Citronensäure im Getränk, um sie zur Genesung überzuführen.

Bei den Entzündungen der parenchymatösen Organe und serösen Häute (Pneumonie, Peritonitis, Pericarditis etc.) im Reactionsstadium sind wir den allgemeinen Indicationen bei diesen Krankheiten gefolgt. Aber der Verlauf dieser Complicationen 
hängt, wie wir wissen, von dem Allgemeinbefinden der Patienten ab. Eine einseitige Pneumonie von nicht allzugrossem Umfange restituirt sich mit Leichtigkeit unter der gewöhnlichen Behandlung, sobald die Urinsecretion reichlich vorhanden und der sonstige Stand der Krankheit günstig ist; im entgegengesetzten Falle haben wir keine Heilung erzielen können. -

Berlin, Anfangs Januar 1867.

\section{XVI.}

\section{Chemisch-mikroskopiscle Beobachtungen aus dem städtisclien Clolera-Lazareth No. III in Berlin.}

Von Max Bruberger.

Kurz nach Beginn der Choleraepidemie des vorigen Jahres war ich so glücklich an dem städtischen Lazareth No. 3. die Stelle eines Assistenzarztes zu erhalten; die Munificenz der städtischen Behörden hatte mir auf Anregung des Hrn. Dr. W. Kühne zur Bearbeitung des reichen Materials noch ein kleines chemisches Laboratorium zur Verfügung gestellt. - Die Fragen, deren Beantwortung ich mir, mit solchen Hülfsmitteln ausgerüstêt und durch den freundlichen Rath des Hrn. Dr. W. Kühne und des Dirig. Hrn. Dr. Goldb a um darauf geführt, vorgenommen hatte, waren: Feststellung des Verbältnisses zwischen den Ausgaben des Organismus an Flüssigkeitsmenge und den Einnahmen desselben. Beantwortung der Fragen, ob, wie oft und in welchen Stadien der Krankheit sich Darmepithel in den Dejectionen nachweisen lasse und welches die chemische Constitution der Choleraexcrete sei. Hrn. Dr. Goldb a um, dem dirigirenden Oberarzte des Lazareths, welcher mir das Material zur zusammenhängenden Bearbeitung überlassen, bin ich zu lebhaftestem Danke verpflichtet. Nachstehende Arbeit ist das Resultat einer dreimonatlichen Beobachtungszeit, ich habe darin versucht, mich möglichst objectiv zu halten und nur Thatsächliches zu geben. 